\title{
TRAVELING TWO AND THREE DIMENSIONAL CAPILLARY GRAVITY WATER WAVES*
}

\author{
WALTER CRAIG ${ }^{\dagger}$ AND DAVID P. NICHOLLS $\ddagger$
}

\begin{abstract}
The main results of this paper are existence theorems for traveling gravity and capillary gravity water waves in two dimensions, and capillary gravity water waves in three dimensions, for any periodic fundamental domain. This is a problem in bifurcation theory, yielding curves in the two dimensional case and bifurcation surfaces in the three dimensional case. In order to address the presence of resonances, the proof is based on a variational formulation and a topological argument, which is related to the resonant Lyapunov center theorem.
\end{abstract}

Key words. water waves, bifurcation theory, traveling waves

AMS subject classifications. $35 ; 76$

PII. S0036141099354181

1. Introduction. Nonlinear periodic traveling waves on the free surface of an ideal fluid tend to form hexagonal patterns. This phenomenon is the focus of a number of recent papers on the subject of water waves, and it is the topic of the present article. In previous work, various approximations to the evolution equations for free surface waves are used, in particular the KP system by J. Hammack, N. Scheffner, and H. Segur [11], and J. Hammack, D. McCallister, N. Scheffner, and H. Segur [12], and alternatively with certain formal shallow water expansions of the Euler equations by P. Milewski and J.B. Keller [16]. A natural question is whether similar patterns can be shown to occur in solutions of the full Euler equations themselves. This is the focus of a series of papers by the present authors. In [18] and in [20] we report on hexagonal wave patterns and other phenomena in numerical computations of solutions, which are shown to satisfy spectral criteria for numerical convergence to solutions of Euler's equations. In the present article we describe rigorous existence results for periodic traveling wave solutions in free surfaces. The goal is to prove the existence of nontrivial traveling wave solutions to the water wave problem for gravity and capillary gravity waves in two and three dimensions. In two dimensions this is proven for both gravity and capillary gravity water waves, constituting a new and relatively straightforward approach to the theorems of T. Levi-Civita and D. Struik. In three dimensions we prove the existence of traveling capillary gravity water waves. However, the problem of gravity waves in three dimensions exhibits the phenomena of small divisors, and it remains open. The theorem that we prove is given below.

THEOREM 1.1. For any spatial period there exist nontrivial periodic traveling wave solutions of the water wave problem in two dimensions for gravity and capillary gravity waves, both in deep water and in water of finite depth. In three dimensions, for any periodic fundamental domain of $\mathbb{R}^{2}$ there exist nontrivial periodic traveling wave solutions of the water wave problem for capillary gravity waves in infinite depth

${ }^{*}$ Received by the editors March 26, 1999; accepted for publication (in revised form) October 26, 1999; published electronically July 5, 2000.

http://www.siam.org/journals/sima/32-2/35418.html

${ }^{\dagger}$ Department of Mathematics, Brown University, Providence, RI 02912 (craigw@math. brown.edu). This author was supported by the NSF under grant DMS 9706273.

${ }^{\ddagger}$ Department of Mathematics, University of Minnesota, Minneapolis, MN 55455 (nicholls@math. umn.edu). This author was partially supported by the Division of Applied Mathematics and the Department of Mathematics at Brown University. 
water and for water of finite depth.

We show that in fact there are many small amplitude traveling waves bifurcating from uniform flow. A solution of the two dimensional problem is of course also a three dimensional one which is constant in one independent variable. In contrast, for the three dimensional problem, the solutions asserted by this theorem explicitly give fully three dimensional wave patterns, as we will see below.

The early rigorous theorems on traveling water waves concern the two dimensional gravity wave problem, by T. Levi-Civita (1925, [15]) in an infinitely deep fluid, and with similar complex variable techniques by D. Struik $(1926,[27])$ for a fluid of finite depth. When surface tension effects are included, the analogous two dimensional traveling wave problem was addressed by E. Zeidler [31] and H. Beckert and E. Zeidler [2] for large coefficient of surface tension, and J.T. Beale [1], who also allowed for small surface tension under conditions of nonresonance. Later J. Reeder and M. Shinbrot reproduced these results [24], and M. Jones and J. Toland [13], [14] dealt specifically with the occurrence of resonance.

The only papers of which we are aware ${ }^{1}$ that give a rigorous analysis of the three dimensional water wave problem are by J. Reeder and M. Shinbrot [23] and T.Y. Sun [28], in which they prove an existence theorem for periodic traveling capillary gravity waves, whose fundamental domain is a "symmetric diamond." In both papers the authors remark that the three dimensional gravity wave problem is an example of a small denominator problem. The proof in [23] applies to most choices of the parameters of the problem (the spatial periods, the acceleration of gravity $g$, the fluid depth $h$, and the Bond number $\left.\sigma / g h^{2}\right)$. However, they must explicitly avoid cases in which a certain linearized operator has a zero eigenvalue of higher multiplicity. These are situations where more than the generic one or two solutions of the linearized equation have the same phase velocity. It is easily shown that these exceptional cases are common, and that they accumulate in parameter space at zero Bond number. The paper [28] also is restricted in the same way, but is in some ways more general as it also contains results for the case of a pressure disturbance applied to the free surface traveling at the phase velocity. The paper [23] also reproves the two dimensional theorems for gravity and for capillary gravity free surface waves, again with the proviso that the parameters are chosen so that a higher multiplicity null eigenvalue is avoided. The two dimensional case without such proviso appears in [13], [14]. With regard to the three dimensional water wave problem without surface tension, see P. Plotnikov [21].

In this paper our proof is based on a reformulation of the capillary gravity wave problem involving surface integrals, and a variational argument which is similar to that used by A. Weinstein [29] and J. Moser [17] in their work on the resonant Lyapunov center theorem. A higher dimensional null space in the free surface problem compares formally with a case of resonance near an elliptic stationary point of a Hamiltonian system. Furthermore, the water wave problem is Hamiltonian in our chosen coordinates, a fact due initially to V.E. Zakharov [30], and this plays a role in a counting argument via an equivariant cohomological index for a lower bound on the number of distinct solutions. Our approach is bifurcation theoretic, with the novelty that for the three dimensional problem the basic parameter is the two dimensional phase velocity, and solutions occur correspondingly in bifurcation surfaces rather than curves.

The organization of the paper is as follows. In section 2 we introduce the

\footnotetext{
${ }^{1}$ We have, however, recently received a preprint of the work of M.D. Groves and A. Mielke [10] on three dimensional capillary gravity waves in channels.
} 
Dirichlet-Neumann operator, reformulate the water wave problem in a set of coordinates involving surface integrals which we have found useful [8], [19], and outline the method of Lyapunov-Schmidt. In section 3 we solve the first Lyapunov-Schmidt equation via the implicit function theorem, and in section 4 we pose the problem as a variational problem for the extrema of an action integral. We then reduce the variational problem to a finite dimensional one using the results of section 3 , and analyze its set of solutions. Finally, in section 5 we prove the analyticity of the DirichletNeumann operator in appropriate function spaces. In many ways this analysis is the heart of the proof. The method is very similar to the one of W. Craig, U. Schanz, and C. Sulem [7] and D. Nicholls [19]; however, we modify it in a nontrivial way to yield the particular estimates which we require.

2. Formulation of the water wave problem. The water wave problem describes the evolution of an ideal fluid with free surface under the effects of gravity (gravity waves) or gravity and surface tension (capillary gravity waves). An ideal fluid is one which is inviscid, incompressible, and irrotational. To begin we will consider an $n$ dimensional fluid, meaning $n-1$ horizontal dimensions and one vertical dimension, and specialize to two and three dimensions later.

2.1. Classical equations and a surface integral formulation. Consider a fluid region given by

$$
S_{\eta}=\left\{(x, y) \in \mathbb{R}^{n-1} \times \mathbb{R} \mid-h \leq y \leq \eta(x, t)\right\},
$$

where $\eta(x, t)$ is the free surface, and $0<h \leq+\infty$. It is well known for an ideal fluid that inside the fluid domain $S_{\eta}$ the fluid velocity $\mathbf{u}$ can be expressed as

$$
\mathbf{u}=\nabla \varphi,
$$

since the fluid is irrotational, and further

$$
\operatorname{div}[\mathbf{u}]=\operatorname{div}[\nabla \varphi]=\Delta \varphi=0,
$$

since the fluid is incompressible. For the problem of water of finite depth the boundary conditions are given by

$$
\begin{array}{cl}
\partial_{y} \varphi=0 & \text { at } y=-h, \\
\partial_{t} \eta+\nabla_{x} \varphi \cdot \nabla_{x} \eta-\partial_{y} \varphi=0 & \text { at } y=\eta(x, t), \\
\partial_{t} \varphi+\frac{1}{2}|\nabla \varphi|^{2}+g \eta-\sigma \operatorname{div}\left[\frac{\nabla_{x} \eta}{\sqrt{1+\left|\nabla_{x} \eta\right|^{2}}}\right]=0 & \text { at } y=\eta(x, t)
\end{array}
$$

where in appropriate units the density of the fluid is taken to be one, $g$ is the acceleration of gravity, and $\sigma / g h^{2}$ is the Bond number of the interface. To consider water of infinite depth we can replace (2.4a) with the following condition:

$$
\partial_{y} \varphi \rightarrow 0 \quad \text { as } y \rightarrow-\infty .
$$

The horizontal boundary conditions will be periodic, which is to say that in two dimensions our surface is parameterized by a circle, and in three dimensions by a torus; both of these are determined by a lattice $\Gamma \subseteq \mathbb{R}^{n-1}$, generated by a nonsingular matrix $A \in \mathbb{R}^{(n-1) \times(n-1)}$ acting on vectors $j \in \mathbb{Z}^{n-1}$, which in turn has a conjugate 
lattice $\Gamma^{\prime} \subseteq \mathbb{R}^{n-1}$ generated by the matrix $2 \pi\left(A^{T}\right)^{-1}$. We recall that a well-behaved function $f$ on the torus $T(\Gamma)=\mathbb{R}^{n-1} / \Gamma$ can be written in terms of its Fourier series

$$
f(x)=\sum_{k \in \Gamma^{\prime}} \hat{f}(k) e^{i k \cdot x}
$$

The classical formulation of the water wave problem is given in (2.3), (2.4a) (or (2.4d)), (2.4b), (2.4c) with periodic boundary conditions. We will follow [8], [25], [19], [18] and recast these equations in a surface integral formulation of the water wave problem. We begin with the observation that when the free surface $\eta(x, t)$ and Dirichlet data at the free surface $\xi(x, t)=\varphi(x, \eta(x, t), t)$ are specified, we can in principle solve the full problem, since $\varphi$ satisfies Laplace's equation with appropriate boundary conditions. In this way the water wave problem is reduced from one posed inside the entire fluid to one posed at the free surface alone. V.E. Zakharov [30] noted this and also made the elegant statement that the surface variables $\eta$ and $\xi$ are canonically conjugate variables with which one may formulate the water wave problem as a Hamiltonian system, with Hamiltonian

$$
H=\int_{T(\Gamma)} \int_{-h}^{\eta} \frac{1}{2}|\nabla \varphi|^{2} d y+\frac{1}{2} g \eta^{2}+\sigma\left(\sqrt{1+\left|\nabla_{x} \eta\right|^{2}}-1\right) d x .
$$

Of course, the full dependence on $\eta$ and $\xi$ is complicated and not explicit. However, W. Craig and C. Sulem [8] found a more convenient representation of the Hamiltonian by noting that the term $\int_{T(\Gamma)} \int_{-h}^{\eta}|\nabla \varphi|^{2} d y d x$ is a quadratic form in the quantity $\xi$ involving the Dirichlet-Neumann operator.

Definition 2.1. The Dirichlet-Neumann operator of the free surface is defined by

$$
G(\eta) \xi=\left.\nabla \varphi\right|_{y=\eta} \cdot N_{\eta},
$$

where the potential function $\varphi$ satisfies (2.3), (2.4a), $\varphi(x, \eta(x))=\xi(x)$, and $N_{\eta}=$ $\left(-\nabla_{x} \eta, 1\right)^{T}$ is a (nonnormalized) exterior normal.

This operator is the central tool in our analysis and a deep understanding of its properties is key to the boundary integral formulation. For our purposes its analyticity in appropriate function spaces is the relevant property which we will state and prove in section 5. Using the Dirichlet-Neumann operator and the divergence theorem, the Hamiltonian for the water wave problem can be written as

$$
H=\int_{T(\Gamma)} \frac{1}{2} \xi(G(\eta) \xi)+\frac{1}{2} g \eta^{2}+\sigma\left(\sqrt{1+\left|\nabla_{x} \eta\right|^{2}}-1\right) d x
$$

By taking the appropriate variations and interpreting them in the correct fashion, or simply taking $(2.4 \mathrm{~b}),(2.4 \mathrm{c})$ and substituting in the Dirichlet-Neumann operator in the appropriate way, one arrives at the following formulation of the water wave problem [5], [7]:

$$
\begin{aligned}
\partial_{t} \eta= & G(\eta) \xi \\
\partial_{t} \xi= & -g \eta-\frac{1}{2\left(1+\left|\nabla_{x} \eta\right|^{2}\right)}\left[\left|\nabla_{x} \xi\right|^{2}-(G(\eta) \xi)^{2}\right. \\
& \left.-2(G(\eta) \xi) \nabla_{x} \xi \cdot \nabla_{x} \eta+\left|\nabla_{x} \xi\right|^{2}\left|\nabla_{x} \eta\right|^{2}-\left(\nabla_{x} \xi \cdot \nabla_{x} \eta\right)^{2}\right]
\end{aligned}
$$




$$
+\sigma \operatorname{div}\left[\frac{\nabla_{x} \eta}{\sqrt{1+\left|\nabla_{x} \eta\right|^{2}}}\right] .
$$

2.2. A variational principle and the method of Lyapunov-Schmidt. There is a variational formulation, related to the principle of stationary action, whose Euler-Lagrange equations give traveling wave solutions of (2.9). Given the lattice $\Gamma \subseteq \mathbb{R}^{n-1}$, consider a class of mappings from the torus $T(\Gamma)=\mathbb{R}_{x}^{n-1} / \Gamma$ to a phase space $X=\left\{u=(\eta(x), \xi(x))^{T}\right\}$ (the topology will be specified later). For these mappings we define $n-1$ many action functionals

$$
I_{j}(\eta(x), \xi(x))=\int_{T(\Gamma)} \eta(x) \partial_{x_{j}} \xi(x) d x
$$

and as well the (averaged) Hamiltonian $H(\eta(x), \xi(x))$ given in (2.8). The formal variational principle is to fix the values of these $n-1$ actions $I_{j}=a_{j}$, and consider extremal points of the Hamiltonian $H$. If such points existed and were sufficiently regular, they would satisfy the Euler-Lagrange equations

$$
\delta H=\sum_{j=1}^{n-1} c_{j} \delta I_{j},
$$

which are traveling wave solutions of system (2.9) with Lagrange multiplier the phase velocity $c=\left(c_{1}, \ldots, c_{n-1}\right)$. There is a torus action on the phase space $X$ given by $T_{\alpha}(\eta(x), \xi(x))=(\eta(x+\alpha), \xi(x+\alpha))$, under which $I_{j}$ and $H$ are invariant, and in this way every critical point is in fact a member of a torus of critical points in $X$. These considerations are, however, purely formal, the level sets of $I_{j}$ are by no means compact, and without further analytic information about the functionals $I_{j}$ and $H$ this procedure does not give rise to actual solutions of the problem, even in finite dimensional settings such as the Lyapunov center theorem. Nonetheless, a reduction that is related to the method of Lyapunov-Schmidt gives rise to a related finite dimensional variational problem, also invariant with respect to the torus action, whose critical points give traveling wave solutions to (2.9). This procedure is analogous to the resonant Lyapunov center theorem and the reduction by J. Moser [17] of the variational problem to a finite dimensional one which is invariant under a circle action.

In order to set up the problem of traveling surface waves, introduce a frame of reference moving with velocity $c$, and change variables in (2.9). This leads to the definition of the functions

$$
\begin{aligned}
(2.12 \mathrm{a}) F_{1}(\eta, \xi, c)= & g \eta-c \cdot \nabla_{x} \xi+\frac{1}{2\left(1+\left|\nabla_{x} \eta\right|^{2}\right)}\left[\left|\nabla_{x} \xi\right|^{2}-(G(\eta) \xi)^{2}\right. \\
& \left.-2(G(\eta) \xi) \nabla_{x} \xi \cdot \nabla_{x} \eta+\left|\nabla_{x} \xi\right|^{2}\left|\nabla_{x} \eta\right|^{2}-\left(\nabla_{x} \xi \cdot \nabla_{x} \eta\right)^{2}\right] \\
& -\sigma \operatorname{div}\left[\frac{\nabla_{x} \eta}{\sqrt{1+\left|\nabla_{x} \eta\right|^{2}}}\right], \\
\text { (2.12b) } F_{2}(\eta, \xi, c)= & c \cdot \nabla_{x} \eta+G(\eta) \xi
\end{aligned}
$$

with which we will abbreviate the problem of traveling waves for the system (2.9) as $F(\eta, \xi, c)=0$. Let $u=(\eta, \xi)^{T}$ and $F: X \times C \rightarrow Y$, where the Banach spaces $X, C$, 
and $Y$ are to be specified later. A trivial branch of solutions to this problem is given by $(0, c)$ for all $c$, and from this trivial branch we produce a nontrivial bifurcation branch of solutions. We will use the implicit function theorem and hence must understand the linearization of $F$ about the trivial solution $\partial_{u} F(0, c) \equiv A(c)$. If $A(c)$ from $X$ to $Y$ is boundedly invertible for some parameter $c$, then we may refer to the implicit function theorem to obtain a unique solution in a neighborhood of $c$, namely, the trivial one. The possible bifurcation points are those values of $c$ for which $A(c)$ has a zero eigenvalue. Using the decomposition of Lyapunov-Schmidt, let $c_{0}$ be a parameter value for which $A\left(c_{0}\right)$ has a nontrivial null space, and let

$$
\begin{aligned}
X_{1} & =\operatorname{null}\left(A\left(c_{0}\right)\right), \\
Y_{1} & =\operatorname{range}\left(A\left(c_{0}\right)\right),
\end{aligned}
$$

and $X_{2}$ and $Y_{2}$ to be such that $X=X_{1} \oplus X_{2}, Y=Y_{1} \oplus Y_{2}$. Let $P$ be the orthogonal projection of $Y$ onto $Y_{1}$ and $Q=I-P$. The method of Lyapunov-Schmidt replaces the problem $F(u, c)=0$ by the equivalent pair of equations

$$
\begin{aligned}
& P F(v+w, c)=0, \\
& Q F(v+w, c)=0,
\end{aligned}
$$

where $v \in X_{1}$ and $w \in X_{2}$. In cases in which the linearized operator admits certain estimates, the first equation can be solved via the implicit function theorem. In the two dimensional problems, and in the three dimensional gravity capillary wave problem, this can be done. In these cases the second equation turns out to be finite dimensional in character and it will be resolved through a reduced variational problem.

In keeping with the program outlined above we begin by identifying the relevant Banach spaces, $X, C$, and $Y$. We recall the following $L^{2}(T(\Gamma))$ based Sobolev spaces:

$$
H^{s}=\left\{f \in L^{2}(T(\Gamma)) \mid\|f\|_{H^{s}}<\infty\right\},
$$

where

$$
\|f\|_{H^{s}}^{2}=\sum_{k \in \Gamma^{\prime}}\langle k\rangle^{2 s}|\hat{f}(k)|^{2}
$$

and

$$
\langle k\rangle^{2}=1+|k|^{2} .
$$

We also introduce the spaces $H_{0}^{s}=\left\{f \in H^{s} \mid \hat{f}(0)=0\right\}$. With these in mind we prove the following lemma.

Lemma 2.2. Suppose that $s>\frac{n-1}{2}$. If $\sigma=0$, then $F: H^{s+1} \times H^{s+1} \rightarrow H^{s} \times H_{0}^{s}$ is an analytic transformation. If $\sigma>0$, then $F: H^{s+2} \times H^{s+1} \rightarrow H^{s} \times H_{0}^{s}$ is an analytic transformation.

Proof. The proof is straightforward and relies heavily upon two facts: first, the Sobolev inequality for $s>\frac{n-1}{2}$,

$$
\|u v\|_{H^{s}} \leq C\|u\|_{H^{s}}\|v\|_{H^{s}} ;
$$

second is the fact that the Dirichlet-Neumann operator is a bounded linear function as a function of $\xi$ from $H^{s+1} \rightarrow H^{s}$, and an analytic function of $\eta$ for $\eta, \xi \in H^{s+1}$. This assertion will be proven in section 5 . We note that in the case $\sigma>0$ there is a 
second order derivative acting on $\eta$, while in the case $\sigma=0$ there are only first order derivatives acting on $\eta$; this accounts for the two different spaces in the statement of the theorem. The fact that the target space of the second component of $F$ is $H_{0}^{s}$ rather than $H^{s}$ is a result of the following calculation:

$$
\begin{aligned}
\hat{F}_{2}(0) & =\int_{T(\Gamma)} c \cdot \partial_{x} \eta+G(\eta) \xi d x \\
& =\int_{T(\Gamma)}\left(\left.\nabla \varphi\right|_{\eta}\right) \cdot N_{\eta} d x \\
& =\int_{T(\Gamma)} \int_{-h}^{\eta} \Delta \varphi d y d x \\
& =0
\end{aligned}
$$

which is true by the divergence theorem for $w=w(v, c)$ and the fact that $\varphi$ is harmonic. Finally, analyticity is proven by the fact that the Dirichlet-Neumann operator is analytic, and that all other appearances of $\eta$ and $\xi$ are in an analytic fashion.

We note that a corollary of this result is the following.

Corollary 2.3. Suppose that $s>\frac{n-1}{2}$. If $\sigma=0$, then $A(c): H^{s+1} \times H_{0}^{s+1} \rightarrow$ $H^{s} \times H_{0}^{s}$ is bounded. If $\sigma>0$, then $A(c): H^{s+2} \times H_{0}^{s+1} \rightarrow H^{s} \times H_{0}^{s}$ is bounded.

We now set $X=H^{s+2} \times H_{0}^{s+1}$ for the case $\sigma>0, X=H^{s+1} \times H_{0}^{s+1}$ for the case $\sigma=0$, and $Y=H^{s} \times H_{0}^{s}$ in either case. In the case of the two dimensional problem we let $C=\mathbb{R}$, and in the case of the three dimensional problem we let $C=\mathbb{R}^{2}$.

2.3. The linearized operator. At this point we will analyze the problem (2.12) linearized around the trivial solution $(u, c)=(0, c)$. In particular we will restrict ourselves to the cases $n=2$ and $n=3$, and we identify the set of parameters $c \in \mathbb{R}$ (respectively, $c \in \mathbb{R}^{2}$ ) for which $A(c)$ has a nontrivial null space. Linearizing the system $(2.12 \mathrm{a})$ about $(u, c)=(0, c)$ involves the Dirichlet-Neumann operator $G(0)=G_{0}=|D| \tanh (h|D|)$.

THEOREM 2.4. The linearization of $F$ about the trivial solution $(0, c)$ is given by

$$
A(c)=\partial_{u} F(0, c)=\left(\begin{array}{cc}
g-\sigma \Delta_{x} & -c \cdot \nabla_{x} \\
c \cdot \nabla_{x} & G_{0}
\end{array}\right)
$$

Furthermore, given $k \in \Gamma^{\prime}$, the null eigenvalues of $A(c)$ on $X$ occur when $c \in \mathbb{R}^{n-1}$ is such that

$$
\Delta_{\sigma}(c, k)=\left(g+\sigma|k|^{2}\right)|k| \tanh (h|k|)-(c \cdot k)^{2}=0 .
$$

Given $c_{0}$ satisfying (2.18) for some $k_{0} \in \Gamma^{\prime}$, the null space of $A\left(c_{0}\right)$ is even dimensional, and it is generically two dimensional. It is spanned by the eigenfunctions

$$
\begin{aligned}
& v_{k}^{1}(x)=\left(\left(c_{0} \cdot k\right) \cos (k \cdot x),\left(g+\sigma|k|^{2}\right) \sin (k \cdot x)\right)^{T}, \\
& v_{k}^{2}(x)=\left(-\left(c_{0} \cdot k\right) \sin (k \cdot x),\left(g+\sigma|k|^{2}\right) \cos (k \cdot x)\right)^{T}
\end{aligned}
$$

for $k \in \Gamma^{\prime}$ in the set of solutions of (2.18).

Proof. The proof is a straightforward calculation, but the role of the function $\Delta_{\sigma}(c, k)$ perhaps needs some explanation. We will always work in function spaces 
smooth enough to admit Fourier expansions of our functions $\eta(x)$ and $\xi(x)$; therefore

$$
\begin{aligned}
A(c)\left(\begin{array}{c}
\eta(x) \\
\xi(x)
\end{array}\right) & =\sum_{k \in \Gamma^{\prime}}\left(\begin{array}{cc}
g+\sigma|k|^{2} & -i c \cdot k \\
i c \cdot k & |k| \tanh (h|k|)
\end{array}\right)\left(\begin{array}{c}
\hat{\eta}(k) \\
\hat{\xi}(k)
\end{array}\right) e^{i k \cdot x} \\
& =\sum_{k \in \Gamma^{\prime}} \hat{A}_{k}(c)\left(\begin{array}{c}
\hat{\eta}(k) \\
\hat{\xi}(k)
\end{array}\right) e^{i k \cdot x} .
\end{aligned}
$$

The operator $A(c)$ is singular if one of the $2 \times 2$ blocks of $\hat{A}(c)$ is singular, which occurs precisely when the determinant $\Delta_{\sigma}(c, k)$ of the $k$ th $2 \times 2$ block is zero, with null vectors determined by the null vector of the singular $2 \times 2$ blocks. The $2 \times 2$ block $\hat{A}_{0}(c)$ has different character, and for all $c \in \mathbb{R}^{n-1}$ it has the null eigenvector $(0, \hat{\xi}(0))$. It is precisely for this reason that we work in the space $X=H^{s+\rho} \times H_{0}^{s+1}$, so that the one dimensional subspace $\{(\eta, \xi)=\gamma(0, \hat{\xi}(0))\}$ does not contribute to the null space of $A(c)$ on $X$. Regarding the dimension of the null space, if $\hat{A}_{k}(c)$ has a null eigenvector, then so does $\hat{A}_{-k}(c)$; hence the null space of $A(c)$ is even dimensional.

The relation (2.18) describes the dispersion relation between a wave number $k$ and its phase velocity $c_{k}$. The set of solutions of (2.18) can now be described, starting with the two dimensional problem. For $k \in \Gamma^{\prime} \backslash\{0\} \subseteq \mathbb{R}$ fixed, a phase velocity $c=c_{k}$ can always be defined through the relation

$$
c^{2} k^{2}=\left(g+\sigma k^{2}\right) k \tanh (h k) ;
$$

in both cases $\sigma>0$ and $\sigma=0$. Therefore, for any specified spatial period $\Lambda$ there is a phase velocity $c$ such that $\Delta_{\sigma}(c, k)=0$ for $k=2 \pi / \Lambda$, giving a solution to the linearized system with period $\Lambda$. Of course both $\pm k$ correspond to the same phase velocity $c$. It is also possible that for a given $c=c_{k_{0}}$ there is another $k_{1} \in \Gamma^{\prime}$ which satisfies (2.20). Physically this corresponds to the situation where a linear gravity wave of lower wave number has the same phase velocity $c$ as one of higher wave number. This situation can occur only if the Bond number satisfies $\sigma / g h^{2}<1 / 3$, where the relation (2.20) has two roots for choices of $c$ such that $\min _{k}\left\{\left(g+\sigma k^{2}\right) k \tanh (h k)\right\}<c^{2}<g h$. These two roots both satisfy $0 \leq k<\sqrt{g / \sigma}$. The phase velocity $c_{k_{0}}$ is chosen so that one of these roots $k_{0}$ lies in the lattice $\Gamma^{\prime}$. When both roots are in $\Gamma^{\prime}$, there is another linear solution with the specified spatial period, and the null space of $A\left(c_{k_{0}}\right)$ is four dimensional.

The three dimensional case is only a little more complex. Given a dual lattice $\Gamma^{\prime} \in \mathbb{R}^{2}$ and any two generators $k_{1}, k_{2}$ of $\Gamma^{\prime}$, there is always a phase velocity $c_{0} \in \mathbb{R}^{2}$ which will satisfy relation (2.18) for both. Indeed, given $k_{1}$, by the discussion of the previous paragraph we may always choose some $c_{1} \in \mathbb{R}^{2}$ which is parallel to $k_{1}$ such that (2.18) holds. Of course any other $c$ such that $c_{1} \cdot k_{1}=c \cdot k_{1}$ will also do, giving a line of solutions of (2.18) through $c_{1}$ perpendicular to $k_{1}$. The same holds for $k_{2}$, and these two lines cannot be parallel; their meeting point is the common solution that we seek. Given this common phase velocity $c_{0}$, it may be that relation (2.18) is satisfied by other wave numbers $k \in \Gamma^{\prime}$ as well as for $k_{1}$ and $k_{2}$, although this situation is not generic. Indeed the relation (2.18) defines a curve in $\mathbb{R}^{2}$ which intersects $k_{1}$ and $k_{2}$ and possibly other points $k_{3}, k_{4}, \ldots \in \Gamma^{\prime}$. This curve is symmetric about the origin, so the solutions of (2.18) appear in pairs $\pm k_{\ell}$. We will normalize by the choice that $k_{\ell} \cdot c_{0}>0$. Note that by the above discussion the situation $k_{\ell}$ perpendicular to $c_{0}$ does not occur. We have shown the following. 
Proposition 2.5. Given any two generators $k_{1}, k_{2}$ of the lattice $\Gamma^{\prime}$, there is a phase velocity $c_{0}$ satisfying $\Delta_{\sigma}\left(k_{\ell}, c_{0}\right)=0, k_{\ell} \cdot c_{0}>0$ for $\ell=1,2$, and therefore $\operatorname{dim} \operatorname{ker} A\left(c_{0}\right) \geq 4$.

A second preliminary result is as follows.

Proposition 2.6. When $\sigma>0$ and $c_{0} \in \mathbb{R}^{2}$ is fixed, the number of lattice points $k \in \Gamma^{\prime}$ satisfying $\Delta_{\sigma}\left(k, c_{0}\right)=0$ is finite.

Proof. The asymptotic behavior of the dispersion relation is that $\omega(k)^{2}=(g+$ $\left.\sigma|k|^{2}\right)|k| \tanh (h|k|) \sim \sigma|k|^{3}$, while $|c \cdot k|^{2} \leq C|k|^{2}$, and therefore the set of root of (2.18) is bounded. Notice that this finiteness result no longer holds when $\sigma=$ 0 .

This paper does not address the three dimensional case with $\sigma=0$, for the reason that it is a situation in which the phenomenon of small divisors is present. Our analysis depends upon the boundedness properties of the operator $A(c)^{-1}$ between appropriate Banach spaces. When $n \geq 3$ and $\sigma=0$ there is a dense set of values of $c \in \mathbb{R}^{n-1}$ for which $A(c)$ has a nontrivial null space, which can even be infinite dimensional. In general, the invertibility properties of the inverse operator, even projected orthogonally to its null space, change sensitively and discontinuously with respect to $c$. It may be that this problem can be solved with methods related to the Nash-Moser implicit function theorem; however, there is at present no published complete proof. Among other things, these are elements of the program outlined in [21]. To describe the phenomenon of small divisors quantitatively, we give the following result.

THEOREM 2.7. The point spectrum of the operator $A(c)$ consists of the set

$$
\left\{\mu_{ \pm}(k)=\frac{1}{2}(g+|k| \tanh (h|k|)) \pm \frac{1}{2} \sqrt{(g-|k| \tanh (h|k|))^{2}+4(c \cdot k)^{2}}\right\}_{k \in \Gamma^{\prime}}
$$

For every choice of the parameters $g, h$, and $c \in \mathbb{R}^{2} \backslash\{0\}$ this set accumulates at $\mu=0$.

Proof. Under the Fourier transform the operator $A(c)$ is $2 \times 2$ block diagonal, with eigenvalues those of the individual blocks. With $\sigma=0$,

$$
\operatorname{det}\left(\hat{A}(c)_{k}-\mu I\right)=\mu^{2}-(g+|k| \tanh (h|k|)) \mu+\left(g|k| \tanh (h|k|)-(c \cdot k)^{2}\right)
$$

and the roots of this relation are the eigenvalues given above. Small divisors will occur when $\Delta_{0}(c, k)=\operatorname{det} \hat{A}(c)_{k}, k \in \Gamma^{\prime}$ satisfies $\left|\Delta_{0}(c, k)\right|<|k|^{1 / 2}$. In this case we call $k \in \Gamma^{\prime}$ a singular site in the dual lattice. The associated small eigenvalue is

$$
\begin{aligned}
\mu_{-}(k) & =\frac{1}{2}(g+|k| \tanh (h|k|))-\frac{1}{2} \sqrt{(g-|k| \tanh (h|k|))^{2}+4(c \cdot k)^{2}} \\
& =\frac{1}{2}(g+|k| \tanh (h|k|))-\frac{1}{2} \sqrt{(g+|k| \tanh (h|k|))^{2}-4 \Delta_{0}(c, k)} \\
& \sim \frac{\Delta_{0}(c, k)}{(g+|k| \tanh (h|k|))},
\end{aligned}
$$

and the latter quantity is $O\left(|k|^{-1 / 2}\right)$ for large and singular $k \in \Gamma^{\prime}$.

It remains to be shown that there exist singular sites in $\Gamma^{\prime}$ with arbitrarily large norm. For this it suffices to consider the problem for deep water $(h=+\infty)$, since $g|k| \tanh (h|k|)-g|k| \sim O\left(|k| e^{-2 h|k|}\right)$, and thus large singular sites for $h$ infinite are effectively ones for finite $h$. For any $c \in \mathbb{R}^{2} \backslash\{0\}$ choose a sequence $n_{j} \in \Gamma^{\prime}$ which obeys an estimate $\left|c \cdot n_{j}\right|<d_{0}$; this is possible in any lattice. Additionally choose $m=m\left(n_{j}\right) \in \Gamma^{\prime}$ such that $|c \cdot m| \sim O(|c||m|),|m|<d_{0}\left|n_{j}\right|^{1 / 2}$, and $\left|m \cdot n_{j}\right|<O\left(\left|n_{j}\right|\right)$. 
With this

$$
\begin{aligned}
g\left|n_{j}+m\right| & =g\left(\left|n_{j}\right|^{2}+2 n_{j} \cdot m+|m|^{2}\right)^{1 / 2} \\
& =g\left|n_{j}\right|\left(1+O\left(\frac{1}{\left|n_{j}\right|}\right)\right)
\end{aligned}
$$

and

$$
\left(c \cdot\left(n_{j}+m\right)\right)^{2}=(c \cdot m)^{2}+O(|m|) .
$$

For these choices $\left|n_{j}+m\right|=O\left(\left|n_{j}\right|\right)$,

$$
g\left|n_{j}+m\right|-\left(c \cdot\left(n_{j}+m\right)\right)^{2}=g\left|n_{j}\right|-\left((c \cdot m)^{2}+O(|m|)\right),
$$

and by further adjustment of $m \in \Gamma^{\prime}$ by choosing it such that $g\left|n_{j}+m^{\prime}\right|-\left(c \cdot\left(n_{j}+m^{\prime}\right)\right)^{2}$ changes sign for some $m^{\prime}$ adjacent to $m$ (this lies within the above constraints) we can ensure that $|g| n_{j}\left|-(c \cdot m)^{2}\right|<O(|m|)$ itself. This choice gives rise to a sequence of lattice sites $k_{j}=n_{j}+m\left(n_{j}\right)$ such that $\left|k_{j}\right| \rightarrow \infty$ and $\mu_{-}\left(k_{j}\right) \sim O\left(\left|k_{j}\right|^{-1 / 2}\right)$, which is more than enough to prove the statements of the theorem.

We have now identified our function spaces, the types of solutions which we seek, and the possible values of $c$ where such bifurcation branches can be found. We must now solve the two bifurcation equations and we begin with the $P$ equation.

3. Existence of solutions to the $\boldsymbol{P}$ equation. In this section we use the implicit function theorem on Banach spaces to solve the $P$ equation of the method of Lyapunov-Schmidt. We have already established that our map $F: X \times C \rightarrow Y$ is analytic and that the linearized operator $A(c)$ is bounded from $X$ into $Y$. It is clear that the projection $P$, being a bounded operator with finite dimensional null space, will not change either of these properties and so the only condition yet to be satisfied in order to use the implicit function theorem is the boundedness of the inverse operator. In the same way that we consider the range of $A(c)$ by operating by $P$, we also consider $X_{2}$ as our domain by viewing $X_{1}$ as part of the parameter space with $C$. This results in a solution

$$
w=w(v, c)
$$

such that $P F(v+w(v, c), c)=0$. With this in mind it remains to prove the following theorem.

TheOREM 3.1. $\left(P \partial_{w} F(0, c)\right)^{-1}$ is a bounded linear operator from $Y_{1}$ to $X_{2}$.

Proof. We begin by identifying a likely candidate to be the inverse operator. If we considered a value of $c$ such that $\Delta_{\sigma}(c, k) \neq 0$ for all $k \in \Gamma^{\prime}$, then an inverse can be found by inverting the $2 \times 2$ blocks on the Fourier side resulting in

$$
\hat{A}(c)_{k}^{-1}=\frac{1}{\Delta_{\sigma}(c, k)}\left(\begin{array}{cc}
|k| \tanh (h|k|) & i c \cdot k \\
-i c \cdot k & g+\sigma|k|^{2}
\end{array}\right) .
$$

This is well defined for all $k \in \Gamma^{\prime} \backslash\{0\}$. At $k=0, \hat{A}(c)_{k}$ is singular, but since the second component of $F$ has $k=0$ mode equal to zero we can define an inverse in the following way:

$$
\hat{A}(c)_{0}^{-1}\left(\begin{array}{c}
\hat{v}(0) \\
0
\end{array}\right) \equiv\left(\begin{array}{c}
\hat{v}(0) \\
g \\
0
\end{array}\right) .
$$


We break up the rest of the proof into three parts, corresponding to each of the three cases which we are considering, since each requires a slightly different analysis.

We begin by studying the case of two dimensions, $\sigma=0$, and a two dimensional null space. In this case $X=H^{s+1} \times H^{s+1}$. Assume that $\hat{A}\left(c_{0}\right)_{ \pm k_{0}}$ is singular. By the discussion of the previous section, all other $\hat{A}(c)_{k}, k \neq \pm k_{0}$ are nonsingular. Let $\tilde{\Gamma}^{\prime}=\Gamma^{\prime} \backslash\left\{ \pm k_{0}\right\}, \tilde{\Gamma}_{0}^{\prime}=\Gamma^{\prime} \backslash\left\{0, \pm k_{0}\right\}$, and consider the estimate

$$
\begin{aligned}
& \left\|\left(P \partial_{w} F(0, c)\right)^{-1}\left(\begin{array}{l}
u^{1} \\
u^{2}
\end{array}\right)\right\|_{X_{2}}^{2} \\
& =\left|\frac{\hat{u}^{1}(0)}{g}\right|^{2}+\sum_{k \in \tilde{\Gamma}_{0}^{\prime}}\langle k\rangle^{2(s+1)}\left|\frac{|k| \tanh (h|k|) \hat{u}^{1}(k)+i c_{0} k \hat{u}^{2}(k)}{\Delta_{0}\left(c_{0}, k\right)}\right|^{2} \\
& +\sum_{k \in \tilde{\Gamma}_{0}^{\prime}}\langle k\rangle^{2(s+1)}\left|\frac{-i c_{0} k \hat{u}^{1}(k)+g \hat{u}^{2}(k)}{\Delta_{0}\left(c_{0}, k\right)}\right|^{2} \\
& \leq\left|\frac{\hat{u}^{1}(0)}{g}\right|^{2}+2 \sum_{k \in \tilde{\Gamma}_{0}^{\prime}}\langle k\rangle^{2(s+1)} \frac{(|k| \tanh (h|k|))^{2}\left|\hat{u}^{1}(k)\right|^{2}+\left(c_{0} k\right)^{2}\left|\hat{u}^{2}(k)\right|^{2}}{\Delta_{0}\left(c_{0}, k\right)^{2}} \\
& +2 \sum_{k \in \tilde{\Gamma}_{0}^{\prime}}\langle k\rangle^{2(s+1)} \frac{\left(c_{0} k\right)^{2}\left|\hat{u}^{1}(k)\right|^{2}+g^{2}\left|\hat{u}^{2}(k)\right|^{2}}{\Delta_{0}\left(c_{0}, k\right)^{2}} \\
& =\left|\frac{\hat{u}^{1}(0)}{g}\right|^{2}+2 \sum_{k \in \tilde{\Gamma}_{0}^{\prime}}\langle k\rangle^{2 s} \frac{\langle k\rangle^{2}\left[(|k| \tanh (h|k|))^{2}\left|\hat{u}^{1}(k)\right|^{2}+\left(c_{0} k\right)^{2}\left|\hat{u}^{2}(k)\right|^{2}\right]}{\Delta_{0}\left(c_{0}, k\right)^{2}} \\
& +2 \sum_{k \in \tilde{\Gamma}_{0}^{\prime}}\langle k\rangle^{2 s} \frac{\langle k\rangle^{2}\left[\left(c_{0} k\right)^{2}\left|\hat{u}^{1}(k)\right|^{2}+g^{2}\left|\hat{u}^{2}(k)\right|^{2}\right]}{\Delta_{0}\left(c_{0}, k\right)^{2}} .
\end{aligned}
$$

Now, by using the fact that we can bound $\Delta_{0}\left(c_{0}, k\right)$ from below by $K_{1}+K_{2}\langle k\rangle^{2}$ we continue the estimate,

$$
\begin{aligned}
& \left\|\left(P \partial_{w} F(0, c)\right)^{-1}\left(\begin{array}{c}
u^{1} \\
u^{2}
\end{array}\right)\right\|_{X_{2}}^{2} \\
& \leq\left|\frac{\hat{u}^{1}(0)}{g}\right|^{2}+C_{1} \sum_{k \in \tilde{\Gamma}_{0}^{\prime}}\langle k\rangle^{2 s}\left|\hat{u}^{1}(k)\right|^{2}+C_{2} \sum_{k \in \tilde{\Gamma}_{0}^{\prime}}\langle k\rangle^{2 s}\left|\hat{u}^{2}(k)\right|^{2} \\
& \leq C\left[\sum_{k \in \tilde{\Gamma}^{\prime}}\langle k\rangle^{2 s}\left|\hat{u}^{1}(k)\right|^{2}+\sum_{k \in \tilde{\Gamma}_{0}^{\prime}}\langle k\rangle^{2 s}\left|\hat{u}^{2}(k)\right|^{2}\right] \\
& =C\left\|\left(\begin{array}{c}
u^{1} \\
u^{2}
\end{array}\right)\right\|_{Y_{1}}^{2} .
\end{aligned}
$$

The case of $\sigma>0$ in two dimensions is very similar, and proceeding in the same way as before we can produce an estimate of the form

$$
\left\|\left(P \partial_{u_{2}} F(0, c)\right)^{-1}\left(\begin{array}{c}
u^{1} \\
u^{2}
\end{array}\right)\right\|_{X_{2}}^{2}
$$




$$
\begin{aligned}
\leq & \left|\frac{\hat{u}^{1}(0)}{g}\right|^{2}+2 \sum_{k \in \tilde{\Gamma}_{0}^{\prime}}\langle k\rangle^{2 s} \frac{\langle k\rangle^{4}\left[(|k| \tanh (h|k|))^{2}\left|\hat{u}^{1}(k)\right|^{2}+\left(c_{0} k\right)^{2}\left|\hat{u}^{2}(k)\right|^{2}\right]}{\Delta_{\sigma}\left(c_{0}, k\right)^{2}} \\
& +2 \sum_{k \in \tilde{\Gamma}_{0}^{\prime}}\langle k\rangle^{2 s} \frac{\langle k\rangle^{2}\left[\left(c_{0} k\right)^{2}\left|\hat{u}^{1}(k)\right|^{2}+\left(g+\sigma|k|^{2}\right)^{2}\left|\hat{u}^{2}(k)\right|^{2}\right]}{\Delta_{\sigma}\left(c_{0}, k\right)^{2}} .
\end{aligned}
$$

Now using the fact that we can produce constants $K_{3}$ and $K_{4}$ such that $\left|\Delta_{\sigma}\right| \geq$ $K_{3}+K_{4}\langle k\rangle^{3}$ we again obtain the estimate desired. The proofs in the cases of higher dimensional null spaces and three dimensions $(\sigma>0)$ are almost the same as the previous except that one has to account for a possibly larger null space for the linearized operator $A(c)$. The sums are over $\tilde{\Gamma}_{0}^{\prime}=\Gamma^{\prime} \backslash\left\{0, \pm k_{j}\right\}_{j=1}^{N}$, which serves to omit from them any of the wave numbers $k_{j}$ such that $\Delta_{\sigma}\left(c, k_{j}\right)=0$. Again, the constants $K_{3}$ and $K_{4}$ can be produced due to the fact that for $|k|$ large enough, $\Delta_{\sigma}(c, k)>$ 0 .

With these estimates in hand it is easy to prove the following theorem.

TheOREM 3.2. The equation $P F(v+w, c)=0$ has a solution

$$
w=w(v, c)
$$

for all $(v, c)$ in a ball $B_{\varepsilon}\left(0, c_{0}\right)$ which is locally unique, such that

$$
\begin{aligned}
P F(v+w(v, c), c) & =0, \\
w(0, c) & =0 .
\end{aligned}
$$

Furthermore, $w$ is analytic as a function of $(v, c),\|w(v, c)\|_{X} \leq C\|v\|_{X}^{2}$, and it is equivariant under the torus action $T_{\alpha} w(v, c)=w\left(T_{\alpha} v, c\right)$.

Proof. We use the implicit function theorem in conjunction with Theorem 3.1.

4. The reduced variational problem. With the choice of $u=v+w(v, c)$ (2.14a) is solved, and we focus on solutions $(v, c)$ which will also result in a solution of $(2.14 \mathrm{~b})$. The approach that we use is close to that of J. Moser [17], with the novelty that the problem is equivariant with respect to the action of a torus $\mathbb{T}^{2}$. The variational problem that we solve is equivalent to finding critical points of the Hamiltonian function $H(u)$ of $(2.8)$, when restricted to the subset of phase space $\left\{u \in X: I_{1}(u)=a_{1}, I_{2}(u)=a_{2}\right\}$. This is not feasible by direct variational methods. Instead, following [17], we pose a reduced variational problem in the finite dimensional space $X_{1}$, whose solutions will solve $(2.14 \mathrm{~b})$. In preparation for this we make an astute choice of $c=c(v)$. The function $u=v+w(v, c)$ solves (2.14a); therefore

$$
(\delta H-c \cdot \delta I)(v+w(v, c))=q(v, c) \in Y_{2} .
$$

Let $\left\{ \pm k_{1}, \ldots \pm k_{N}\right\}=K \subseteq \Gamma^{\prime}$ be the collection of wave numbers for which $\Delta\left(c_{0}, k_{p}\right)=$ 0 , which are normalized so that $c_{0} \cdot k_{p}>0$. We also choose the norm $|v|$ so that it is invariant under the torus action $v \rightarrow T_{\alpha} v$.

Lemma 4.1. Suppose that the vector $a=\left(a_{1}, a_{2}\right)$ is not collinear with any $k_{p} \in K$, and that $\left|\left(a_{1}, a_{2}\right)\right|<\delta$ for sufficiently small $\delta$. On the set $\left\{(v, c): I_{1}(v+w(v, c))=\right.$ $\left.a_{1}, I_{2}(v+w(v, c))=a_{2}\right\} \subseteq X_{1} \times \mathbb{R}^{2}$ we can make a choice of $c=c(v)$ to satisfy

$$
\int_{T(\Gamma)}\left(q(v, c), \delta_{u} I_{j}(v+w(v, c))\right) d x=0, \quad j=1,2 .
$$


Furthermore, $c(v)$ is invariant under the action $T_{\alpha}$ of the torus, $c\left(T_{\alpha} v\right)=c(v) ; \mid c(v)-$ $c_{0} \mid=O\left(|v|^{2}\right)$; and for $v \neq 0 c(v)$ is real analytic in $v$.

We will defer the proof of this for several pages, for the sake of continuity of the argument. Using both $c(v)$ and $w(v, c(v))$, define the functionals

$$
I_{j}^{Q}(v)=I_{j}(v+w(v, c(v))), \quad j=1,2,
$$

and

$$
H^{Q}(v)=H(v+w(v, c(v))) .
$$

These functionals are invariant under the torus action $T_{\alpha}: X_{1} \rightarrow X_{1}$. For $a=\left(a_{1}, a_{2}\right)$ not collinear with any of the set $K$, and for $|a|<\delta$ sufficiently small, consider the subset of the space $X_{1}$

$$
S(a)=\left\{v \in X_{1}: I_{1}^{Q}(v)=a_{1}, I_{2}^{Q}(v)=a_{2}\right\} .
$$

Clearly $S(a)$ is also invariant under $T_{\alpha}$, and it is a smooth submanifold of $X_{1}$, to which Lemma 4.1 applies. Finally define the reduced action functional

$$
A(v)=H^{Q}(v)-c(v) \cdot\left(I^{Q}(v)-a\right) .
$$

THEOREM 4.2. When $\delta_{v} I_{1}^{Q}$ and $\delta_{v} I_{2}^{Q}$ are linearily independent at all $v \in S(a)$, then $S(a)$ is a submanifold of $X_{1}$ of codimension 2. In this case, critical points of $A(v)$ on $S(a)$ correspond to solutions $u=v+w(v, c(v)), c(v)$ of the bifurcation equation $Q F=0$ of $(2.14 \mathrm{~b})$.

Proof. Choices of $(v, c)$ such that $q(v, c)=0$ correspond precisely to solutions of the desired equation (2.14b). The choice of $c(v)$ prescribed in Lemma 4.1 is so that $q$ is orthogonal in $X_{1}$ to the subspace of normal vectors $N_{v}(S(a))$. Indeed with $q \in X_{1}$, and a basis of $N_{v}(S(a))$ given by $\left\{\delta_{v} I_{1}^{Q}, \delta_{v} I_{2}^{Q}\right\}$,

$$
\begin{aligned}
\int_{T(\Gamma)}\left(q, \delta_{v} I_{j}^{Q}\right) d x & =\int_{T(\Gamma)}\left(q, Q \delta_{u} I_{j}(v+w(v, c))\right) d x \\
& =\int_{T(\Gamma)}\left(q, \delta_{u} I_{j}\right) d x \\
& =0 .
\end{aligned}
$$

Now suppose that $v$ is a critical point on $S(a)$ of the functional $A(v)$. For any $\delta v \in T_{v}(S(a))$ the tangent space, we have the calculation

$$
\begin{aligned}
\partial_{v} A(v) \cdot \delta v= & \int_{T(\Gamma)} Q\left(\delta_{u} H(v+w)-c(v) \cdot \delta_{u} I(v+w)\right) \delta v d x \\
& +\int_{T(\Gamma)} P\left(\delta_{u} H(v+w)-c(v) \cdot \delta_{u} I(v+w)\right)\left(\partial_{v} w \cdot \delta v\right) d x \\
= & 0
\end{aligned}
$$

where the term containing $\partial_{v} c(v)$ has dropped out as the expression is evaluated on $S(a)$. Furthermore $\left(\partial_{v} w \cdot \delta v\right) \in X_{2}$, and we have resolved the first bifurcation equation $(2.14 \mathrm{a})$, so that the second term is zero as well. Thus for $v \in S(a)$ and $\delta v \in T_{v}(S(a))$, $\partial_{v} A(v) \cdot \delta v=\int_{T(\Gamma)}(q, \delta v) d x$, and at critical points it vanishes. Since $c=c(v)$ is chosen so that $\int_{T(\Gamma)}(q, \delta v) d x=0$ for all $\delta v \perp T_{v}(S(a))$ as well, indeed critical points 
correspond to zeros of $q=q(v, c(v))$. Because $A(v)$ is invariant under the action $T_{\alpha}$, so is the set of critical points on $S(a)$.

At this point in the argument, any choices of $a$ such that $S(a)$ is compact will yield at least one solution of the Euler equations $(2.9)(S(a)$ is a point when $m=2)$. We will, however, be able to use the topology of the quotient space $S(a) / \mathbb{T}^{2}$ to obtain a more precise and generally larger lower bound on the number of critical orbits of $A(v)$ on $S(a)$.

4.1. Application of a $T^{2}$ equivariant cohomological index. Parameterize $v \in X_{1}$ by $\mathbb{C}^{N}$ by setting $z=\left(r_{1} e^{i \varphi_{1}}, \ldots, r_{N} e^{i \varphi_{N}}\right)$, and

$$
v=\sum_{p=1}^{N} r_{p}\left(\cos \left(\varphi_{p}\right) v_{k_{p}}^{1}+\sin \left(\varphi_{p}\right) v_{k_{p}}^{2}\right) .
$$

The torus action $T_{\alpha}$ on $X_{1}$ is defined by

$$
T_{\alpha} v=\sum_{p=1}^{N} r_{p}\left(\cos \left(\varphi_{p}+k_{p} \cdot \alpha\right) v_{k_{p}}^{1}+\sin \left(\varphi_{p}+k_{p} \cdot \alpha\right) v_{k_{p}}^{2}\right),
$$

which in $\mathbb{C}^{N}$ is efficiently described as $T_{\alpha} z=\left(r_{p} e^{\left(i \varphi_{p}+k_{p} \cdot \alpha\right)}\right)_{p=1}^{N}$. A calculation yields that

$$
I_{j}^{Q}(v)=\frac{1}{2} \int_{T(\Gamma)}\left(v, J \partial_{x_{j}} v\right) d x=\sum_{p=1}^{N} k_{p}^{j} r_{p}^{2}\left(c_{0} \cdot k_{p}\left(g+\sigma\left|k_{p}\right|^{2}\right)\right)|T(\Gamma)|
$$

for $j=1,2$ where $J=\left(\begin{array}{cc}0 & 1 \\ -1 & 0\end{array}\right)$. Note that we have previously normalized wave numbers so that $c_{0} \cdot k_{p}>0,1 \leq p \leq N$. We will use the notation $c(p)=c_{0} \cdot k_{p}(g+$ $\left.\sigma\left|k_{p}\right|^{2}\right)|T(\Gamma)|$.

Let us start the discussion with the nonresonant case $N=2$, with $k_{1}, k_{2} \in \Gamma^{\prime}$ not collinear. Choose a $2 \times 2$ change of basis $M$ so that $M k_{1}=(1,0)^{T}$ and $M k_{2}=(0,1)^{T}$. This gives an equivalent definition of $S(a)=\left\{v \in X_{1}: M I=M a=b\right\}$ and modifies the torus action to a product action $T_{\beta} z=\left(z_{1} e^{i \beta_{1}}, z_{2} e^{i \beta_{2}}\right)$, where $M^{T} \beta=\alpha$. Clearly $S(a)$ is the two dimensional torus

$$
S(a)=\left\{v: r_{1}^{2} c^{\prime}(1)=b_{1}, r_{2}^{2} c^{\prime}(1)=b_{2}\right\}
$$

for positive constants $c^{\prime}(p)=\sum_{q} M_{p q} c(q)$, and the orbit under $T_{\beta}$ of any point of $S(a)$ consists of the whole set. Therefore the Hamiltonian $H$ is constant on the set, and we have proved the following result.

THEOREM 4.3. In case $N=2$ and $k_{1}, k_{2}$ not collinear, whenever a is not collinear with either $k_{1}$ or $k_{2}$, and $|a|<\delta$, each submanifold $S(a) \in X_{1}$ corresponds to a solution of (2.9) and its translates by $T_{\alpha}$. Furthermore the family of solutions $u=v+$ $w(v, c(v)) \in X, v \neq 0$, is locally real analytic, forming a four dimensional bifurcation manifold which is invariant under the action of $T_{\alpha}$.

The theorem is the analogue of the original Lyapunov center theorem, in which there are no allowances for resonances. The statement of analyticity is an immediate consequence of the regularity results of Theorem 3.2 and Lemma 4.1, stemming ultimately from the implicit function theorem. In the particular case that $k_{1}=\left(k_{1}^{1}, k_{1}^{2}\right)$, and $k_{2}=\left(k_{1}^{1},-k_{1}^{2}\right)$, with $k_{1}^{1} \neq k_{1}^{2}$, the curve $\left\{a_{1}=a_{2}\right\} \in S(a)$ consists of the symmetric diamond solutions of the three dimensional water wave problem in J. Reeder and M. Shinbrot [23]. 
Now turn to the resonant case $N \geq 2$. We will consider two possibilities separately, either (i) $N>2$, for which necessarily there are two noncollinear wave vectors among $\left\{k_{p}\right\}_{p=1}^{N}$, or else (ii) $N=2$ and $k_{1}$ and $k_{2}$ are linearly dependent. The latter case corresponds to the two dimensional resonant problem, with resonant interaction between periodic gravity waves and capillary waves with the same phase velocity; this was previously discussed in M. Jones and J. Toland's work [13], [14]. In case (ii) we will give an independent proof of the existence of at least two distinct periodic solutions; the proof will be put off until the end of the present section.

In case (i) of the preceding paragraph, in which $N>2$, the wave vectors $\left\{k_{p}\right\}_{p=1}^{N}$ involved in the kernel $X_{1}$ lie within the cone $\left\{c_{0} \cdot k>0\right\}$. Choose $k_{1}$ to be the leftmost of this collection, $k_{N}$ to be the rightmost. In case either or both of these are collinear with another from the collection (from our discussion of the dispersion relation in section 2.3, at most two wave vectors can be collinear), take $k_{1}$ and/or $k_{N}$ to be the smaller, and $k_{2}$ and/or $k_{N-1}$ to be the bigger. As in the nonresonant case we may make a change of basis $M$ so that $k_{1}=(1,0)^{T}$ and $k_{N}=(0,1)^{T}$; without changing notation assume that this is so. Then all other wave vectors are expressed as $k_{p}=k_{p}^{1} k_{1}+k_{p}^{2} k_{N}$ for rationals $k_{p}^{\ell}>0$, except that in case of collinearity it may be that $k_{2}=\left(k_{2}^{1}, 0\right)^{T}$ and/or $k_{N-1}=\left(0, k_{N-1}^{2}\right)^{T}$. All of the other coefficients are positive from this exercise, as $k_{p}$ lie in the positive cone of the new basis $\left\{k_{1}, k_{N}\right\}$. We also order the remaining wave vectors $k_{p}=\left(k_{p}^{1}, k_{p}^{2}\right)^{T}$ in terms of increasing slope; $k_{p}^{2} / k_{p}^{1} \leq k_{p+1}^{2} / k_{p+1}^{1}$. It again follows from section 2.3 that at most two wave vectors can have a common slope.

THEOREM 4.4. Suppose that for all $1 \leq p \leq N, k_{p}^{2} / k_{p}^{1} \neq a_{2} / a_{1}$, and that $|a|<\varepsilon^{2}$ for sufficiently small $\varepsilon$. Then the set $S(a)=\left\{v \in X_{1}: I_{1}=a_{1}, I_{2}=a_{2}\right\}$ is a compact submanifold of $X_{1}$.

Proof. From the form (4.9) of the action integrals $I_{j}^{Q}$, it is clear that the gradients $\delta_{v} I_{1}^{Q}$ and $\delta_{v} I_{2}^{Q}$ are independent except when $\left(a_{1}, a_{2}\right)$ is taken so that two semiaxes of the cylindrical ellipsoids $\left\{I_{1}^{Q}=a_{1}\right\}$ and $\left\{I_{2}^{Q}=a_{2}\right\}$ coincide, and we can invoke Theorem 4.2. There is in fact more structure than this. When $a_{2} / a_{1}=k_{p}^{2} / k_{p}^{1}$, and $k_{p}$ is the sole wave vector with this slope, the intersection must be at $\left\{z_{j}=\right.$ $0, j \neq p\} \cap S(a)$, which is a circle (invariant under the action of $T_{\alpha}$ ). In case of multiplicity two, this is at $\left\{z_{j}=0, j \neq p, p+1\right\} \cap S(a)$, which is a $T_{\alpha}$ invariant ellipsoid homeomorphic to $S^{3}$.

With no further work one can see that for $N>2$ and for choices of $a$ avoiding the singular cases $\left\{k_{p}^{2} / k_{p}^{1}: p=1, \ldots, N\right\}$ there are at least two distinct solutions of $(2.9)$, corresponding to the maximum and minimum of the reduced Hamiltonian $H^{Q}$ on $S(a)$. In all cases in which both $a_{1}, a_{2}>0$ these are truly three dimensional solutions, as they have a nonzero component of $v_{p} \in X_{1}$ for at least two linearly independent wave vectors $k_{p}$. Indeed, if $k_{p}$ is the only wave number having slope $k_{p}^{2} / k_{p}^{1}$, and if $a_{2} / a_{1} \neq k_{p}^{2} / k_{p}^{1}$, then $\left\{z_{j}=0: j \neq p\right\} \cap S(a)$ is empty. In case $k_{p}$ is collinear with $k_{p+1}$, but still $a_{2} / a_{1} \neq k_{p}^{2} / k_{p}^{1}$, then as well $\left\{z_{j}=0: j \neq p, p+1\right\} \cap S(a)=\emptyset$, since if $z=\left(0, \ldots z_{p}, z_{p+1}, \ldots, 0\right) \in S(a)$, then

$$
\left|z_{p}\right|^{2}+\frac{k_{p+1}^{1}}{k_{p}^{1}}\left|z_{p+1}\right|^{2}=\frac{a_{1}}{k_{p}^{1}}=\left|z_{p}\right|^{2}+\frac{k_{p+1}^{2}}{k_{p}^{2}}\left|z_{p+1}\right|^{2}=\frac{a_{2}}{k_{p}^{2}},
$$

giving the contradiction $a_{2} / a_{1}=k_{p}^{2} / k_{p}^{1}$. This argument shows that solutions of (2.9) associated with $v \in S(a)$ are genuinely three dimensional in character.

Theorem 4.5. Given $v \in S(a)$ with $a_{2} / a_{1} \notin\left\{k_{p}^{2} / k_{p}^{1}: p=1, \ldots, N\right\}$, then $v$ contains nonzero Fourier modes for at least two wave vectors which are not collinear. 
There is further topology of the orbit space $S(a) / T^{2}$ which will, in cases of resonance $N>2$, guarantee, in general, that there are more solutions. This will be shown by an argument involving a cohomological index equivariant with respect to the group action of a torus, similar to the topological category argument of J. Moser [17] with the action of a circle. In what follows, ind $T_{\alpha}(S)$ will be a $T_{\alpha}$ equivariant cohomological index of the set $S$. The following theorem gives a lower bound for distinct critical orbits of a $T_{\alpha}$ invariant function $H^{Q}$ on $S(a)$ in this situation, in terms of the index. It furthermore provides an estimate of the index, which is sharp.

THEOREM 4.6. Let $N>2$ and choose $a$ as in Theorem 4.4 so that $S(a)$ is a compact manifold. Then there exist at least ind $T_{\alpha}(S(a))+1$ distinct critical orbits of $H^{Q}$ on $S(a)$, corresponding to distinct solutions of (2.9). We can furthermore make a choice of the index so that ind $T_{\alpha}(S(a))=N-2$.

A standard argument implies that the number of critical points of invariant functionals $H$ on the set $S(a)$ is bounded below by ind $T_{\alpha}(S(a))+1$. The crux of the proof is to show that, while the topology of $S(a)$ and $S(a) / T^{2}$ varies depending upon $a$, in all nonsingular cases one can define an index so that ind $T_{\alpha}(S(a))=N-2$.

We claim that for all $a$ such that $a_{2} / a_{1} \notin\left\{k_{p}^{2} / k_{p}^{1}: p=1, \ldots, N\right\}$, then $S(a) \simeq$ $S^{2 d-1} \times S^{2(N-d)-1}$. Indeed, define

$$
J_{1}=\frac{1}{a_{1}} I_{1}+\frac{1}{a_{2}} I_{2}=\sum_{p=1}^{N} c(p)\left(\frac{k_{p}^{1}}{a_{1}}+\frac{k_{p}^{2}}{a_{2}}\right) r_{p}^{2}
$$

and

$$
J_{2}=\frac{1}{a_{1}} I_{1}-\frac{1}{a_{2}} I_{2}=\sum_{p=1}^{N} c(p)\left(\frac{k_{p}^{1}}{a_{1}}-\frac{k_{p}^{2}}{a_{2}}\right) r_{p}^{2} ;
$$

an equivalent description of $S(a)$ is that

$$
S(a)=\left\{v \in X_{1}: J_{1}=2, J_{2}=0\right\} .
$$

Suppose that $k_{d}^{2} / k_{d}^{1}<a_{2} / a_{1}<k_{d+1}^{2} / k_{d+1}^{1}$, then for $1 \leq p \leq d$ the coefficients $\left(k_{p}^{1} / a_{1}-k_{p}^{2} / a_{2}\right)$ of $J_{2}$ are positive, and they are negative for $d+1 \leq p \leq N$. We may write $P=\sum_{p=1}^{d} c(p)\left(\frac{k_{p}^{1}}{a_{1}}-\frac{k_{p}^{2}}{a_{2}}\right) r_{p}^{2}, N=-\sum_{p=d+1}^{N} c(p)\left(\frac{k_{p}^{1}}{a_{1}}-\frac{k_{p}^{2}}{a_{2}}\right) r_{p}^{2}$; then $J_{2}(v)=P-N$ and the set $S(a)$ is homeomorphic through radial projections to the manifold

$$
\begin{aligned}
\tilde{S}(a) & =\left\{z \in \mathbb{C}^{N}:(P+N)(z)=2, \quad(P-N)(z)=0\right\} \\
& =\left\{z \in \mathbb{C}^{N}: P(z)=1 \text { and } N(z)=1\right\} .
\end{aligned}
$$

This expression makes it is clear that $\tilde{S}(a) \simeq S^{2 d-1} \times S^{2(N-d)-1}$, a product of odd dimensional spheres.

We are interested in functions on $S(a)$ which are invariant under the action of $T_{\alpha}$; therefore, it is essentially the index of the orbit space $S(a) / T^{2} \simeq S^{2 d-1} \times$ $S^{2(N-d)-1} / T^{2}$ which is relevant. As a first example, we will suppose that $T_{\alpha}$ is a product $T^{2}$ action on the manifold $S^{2 d-1} \times S^{2(N-d)-1}$, and that it is free (although in the capillary gravity wave problem this is almost never the case);

$$
T_{\alpha}\left(z_{1}, \ldots, z_{N}\right)=\left(z_{p} e^{i \alpha_{1} k^{1}}\right)_{p=1}^{d}\left(z_{p} e^{i \alpha_{2} k^{2}}\right)_{p=d+1}^{N} .
$$

Therefore $S(a)$ is a manifold which factors into two odd dimensional spheres, each with a circle action which is equivalent to the Hopf fibration. That is, the orbit space 
is $S(a) / T^{2} \simeq \mathbb{C} P(d-1) \times \mathbb{C} P(N-d-1)$. In this case we will take the equivariant cohomological index ind $T_{\alpha}(S(a))$ to coincide with the Cech cohomology cuplength of the quotient manifold; cuplength $\left(S(a) / T^{2}\right)$. The following proposition consists of standard facts about the notion of index, Liusternik-Schnirlman category, and cuplength.

Proposition 4.7. The number of critical points of a $C^{2}$ function $H$ on $M$ is bounded below by $\operatorname{Cat}(M)$. Furthermore,

(i) $\operatorname{Cat}(M) \geq$ cuplength $(M)+1 \geq$ ind $_{T_{\alpha}}(M)+1$;

(ii) cuplength $\left(M_{1} \times M_{2}\right)=\operatorname{cuplength}\left(M_{1}\right)+\operatorname{cuplength}\left(M_{2}\right)$;

(iii) when $M \simeq \mathbb{C} P(q)$, then $\operatorname{Cat}(M)=\operatorname{cuplength}(M)+1=q+1$.

We deduce from (ii) and (iii) that

(iv) Cat $(\mathbb{C} P(d-1) \times \mathbb{C} P(N-d-1)) \geq N-1$.

The remaining task is to produce the same lower bound in the general case, in which the torus action $T_{\alpha}$ on $S(a)$ is not free, and when the action of $T_{\alpha}$ is as a twisted product. We note that, while $T_{\alpha}$ is not a free action, at least the stabilizers $s(z)=\left\{\alpha \in T^{2}: T_{\alpha} z=z\right\}$ are always finite. The following argument is an adaptation of the classical one of A. Borel, which we learned from T. Goodwillie and D. Sinha.

The first step in the construction of a $T_{\alpha}$ equivariant cohomology for $S(a)$ is to identify a universal total space $E=S^{\infty} \times S^{\infty}$ and the classifying space $E / T^{2}=$ $B_{T^{2}} \sim \mathbb{C} P^{\infty} \times \mathbb{C} P^{\infty}$; see, for example, E. Fadell and P. Rabinowitz [9] or Rabinowitz [22]. The action of $T_{\alpha}$ on $E \times S(a)$ is free, and because $E$ is contractible and the only isotropy subgroups which appear are finite, the space $S(a) / T^{2}$ and the homotopy orbit space $(E \times S(a)) / T^{2}$ have the same rational cohomology.

The cohomology of the classifying space $B_{T^{2}}$ is generated by two elements $y_{1}=$ $y \otimes 1$ and $y_{2}=1 \otimes y$, where $y$ is the generator of the cohomology of $\mathbb{C} P^{\infty}$. Because of the presence of more than one generator, the construction of [9] will not work directly. We note, however, that in the same vein a well-defined index based on cuplength can be defined for any graded subalgebra of $H^{*}(B ; \mathbb{Q})$. Let $Y$ be a finitely generated graded subalgebra of $H^{*}(B ; \mathbb{Q})$ with a basis $\left\{y_{1}, \ldots, y_{q}\right\}$, and let $F:(E \times S(a)) / T^{2} \rightarrow B_{T^{2}}$ be a classifying map. We define an equivariant cohomological index to be

$$
\operatorname{ind}_{Y}\left((E \times S(a)) / T^{2}\right)=\max \left\{|k|: k \in \mathbb{N}^{q}, F^{*}\left(y^{k}\right) \neq 0\right\} .
$$

This index coincides with that of E. Fadell and P. Rabinowitz [9] when $Y$ is generated by one element, and with the usual cuplength when $Y=H^{*}(B ; \mathbb{Q})$. In our situation we will take $Y$ to be generated by $\left\{y_{1}, y_{2}\right\}$, which is the latter case.

The rational cohomology of the homotopy orbit space $(E \times S(a)) / T^{2}$ is obtained using the Leray-Serre spectral sequences. This calculation can be paraphrased in terms of Poincaré series. The $E_{2}$ page of the spectral sequence is $E_{2}^{p, q}=H^{p}\left(B_{T^{2}}\right) \otimes$ $H^{q}(S(a))$, whose associated Poincaré series is

$$
P_{B}(t)=\left(1-t^{2}\right)^{-2} .
$$

One nontrivial differential occurs at the $E_{2 p}$ page, and another at the $E_{2(N-p)}$ page (or in the inverse order, if $N / 2<p$ ), resulting in the Poincare polynomial for the quotient

$$
\begin{aligned}
P_{(E \times S(a)) / T^{2}}(t) & =\frac{\left(1-t^{2 p}\right)\left(1-t^{2(N-p)}\right)}{\left(1-t^{2}\right)^{2}} \\
& =\left(1+t^{2}+\cdots+t^{2(p-1)}\right)\left(1+t^{2}+\cdots+t^{2(N-p-1)}\right) .
\end{aligned}
$$


The coefficients of $P_{(E \times S(a)) / T^{2}}(t)$ give the Betti numbers $\beta_{j}$ of $S(a) / T^{2}$, as its rational cohomology coincides with that of the homotopy orbit space. From this expression, $\beta_{2(N-2)}=1$; hence $H^{2(N-2)}\left((E \times S(a)) / T^{2} ; \mathbb{Q}\right)$ is one dimensional, and there is some nonzero element in the coset of $\left\{y_{1}^{\gamma} y_{2}^{N-2-\gamma}\right\}_{\gamma=0}^{N-2}$. All higher cohomology classes vanish. We deduce that there are classes $x_{1}, x_{2} \in H^{2}$ for which the cup product $x_{1}^{\gamma} x_{2}^{N-2-\gamma}$ is nonzero, so that ind $T_{\alpha}\left((E \times S(a)) / T^{2}\right)=N-2$. Applying Proposition 4.7 , we conclude the result of Theorem 4.6.

We note for further interest that for Morse functions $H$ on $S(a) / T^{2}$, the Morse inequalities state that the number of critical points is at least $P_{(E \times S(a)) / T^{2}}(1)=$ $\sum_{j=1}^{2(N-2)} \beta_{j}=p(N-p)$. This estimate exceeds $N-1$, strictly so if $p \neq 1, N-1$. The equivariant cohomology of products of spheres under torus actions is relevant in other problems, in particular in the problem of resonant tori in dynamical systems; it is discussed further in W. Craig and D. Haskell [6].

4.2. Two dimensional capillary gravity waves. The point of this short subsection is to provide an alternate proof of the existence of gravity waves in two dimensions [15], [27], and capillary gravity waves in two dimensions, both in the regular case [31], [2], [1] and in the presence of resonance [13], [14]. This can be done in the present framework, considering the circle $T(\Gamma)=\mathbb{R} / \Gamma$ and setting our basic function space to be $X=H^{s}(T(\Gamma))$. The null space of the linearized operator $A$ is denoted by $X_{1}$; in the nonresonant case it is two dimensional, and in the case of resonance it is four dimensional. By Theorem 3.2 we obtain a solution to $(2.14 \mathrm{a})$ in a ball $B_{\varepsilon}\left(0, c_{0}\right)$ which is analytic in both variables $(v, c)$. The relevant functionals for the variational problem are

$$
I_{1}=\int_{S^{1}} \eta \partial_{x_{1}} \xi d x
$$

and $H$ gives as in (2.8) by a one dimensional integral. In the two dimensional problem the group action is a circle action on $S(a)$ which leaves the functions $H, I$ invariant. Furthermore, the one dimensional version of Lemma 4.1 holds [16, Lemma 2, p. 739], giving an analytic function $c=c(v)$ such that $q=F(v+w(v, c(v)), c(v))$ is in the tangent space of $S(a)=\left\{v \in X_{1}: I^{Q}(v)=a\right\}$. The object is to solve (2.14b) using the reduced variational problem.

In the nonresonant case, $N=1$ and each subset $S(a) \in X_{1}, a>0$, is a one dimensional submanifold consisting entirely of solutions of (2.9), related to each other by translation by $T_{\alpha}$; this is the analog of Theorem 4.3. The solutions are in fact analytic in $v$, as in the classical Lyapunov center theorem.

We now consider the resonant case. Let us suppose that the null space of $A\left(c_{0}\right)$ is generated by Fourier modes $k_{1}, k_{2}=R k_{1} \in \Gamma^{\prime} \simeq \mathbb{Z}$. For small Bond number, we have in fact $k_{1} \ll k_{2}$. The subset of $X_{1}$ with fixed momentum is

$$
S(a)=\left\{v \in X_{1} \quad: I_{1}=c(1)\left|z_{1}\right|^{2}+c(2)\left|z_{2}\right|^{2}=a\right\} .
$$

The circle action on $S(a)$ is given by $T_{\alpha} z=\left(z_{1} e^{i \alpha}, z_{2} e^{i R \alpha}\right)$, and incidentally the quotient $S(a) / T_{\alpha}$ is never a manifold. Nonetheless, the submanifold $S(a) \simeq S^{3}$ is compact, and therefore the function $H$ is either constant, or else has at least two distinct critical values, namely, its maximum and its minimum. This gives rise to at least two distinct critical circles of $H$ on $S(a)$, invariant under $T_{\alpha}$.

ThEOREM 4.8. The two dimensional gravity wave problem and the two dimensional capillary gravity wave problem have analytic families of periodic solutions bifurcating from each $c_{k}, k \in \Gamma^{\prime}$, for which the null space $X_{1}$ is two dimensional. In 
case of resonance in the capillary gravity wave problem, $X_{1}$ is four dimensional and there are at least two distinct solutions on every level surface $S(a)$ of the momentum $I_{1}$, for sufficiently small $a>0$.

We remark that we would not obtain a better lower estimate for the number of critical orbits of $H$ on $S(a)$ from an index theory argument, or even from a more refined argument by Morse theory, if indeed we were to show that $H$ was a Morse function on $S(a) / T^{2}$.

4.3. Proof of Lemma 4.1. To finish this section we give a proof of Lemma 4.1, which is based on the implicit function theorem. From Theorem 3.2 we have the estimate $\|w(v, c)\|_{X} \leq C\|v\|_{X}^{2}$, and we also find

$$
\begin{aligned}
\|q\|_{Y}= & \left\|\delta_{u} H(v+w)-c \cdot \delta_{u} I(v+w)\right\|_{Y} \\
\leq & \left\|Q\left(\delta_{u}^{2} H(0)(v+w)-c_{0} \cdot \delta_{u} I(v+w)\right)\right\|_{Y} \\
& +\left\|Q\left(\delta_{u} H_{3}(v+w)-\left(c-c_{0}\right) \cdot \delta_{u} I(v+w)\right)\right\|_{Y} \\
= & \left\|Q\left(\delta_{u} H_{3}(v+w)-\left(c-c_{0}\right) \cdot \delta_{u} I(v)\right)\right\|_{Y} .
\end{aligned}
$$

The notation is that $I=\left(I_{1}, I_{2}\right)$, that $\delta_{u} H(u)=\delta_{u}^{2} H(0) u+\delta_{u} H_{3}(u)$ describes the Taylor series expansion with remainder, and we have used that $Q$ projects orthogonally in $X$ onto an invariant subspace of $J$ (a symplectic subspace) so that $Q \delta_{u} I(u)=$ $\delta_{u} I(Q u)$. Letting $v=\sum_{p=1}^{N} r_{p}\left[\cos \left(\varphi_{p}\right) v_{k_{p}}^{1}+\sin \left(\varphi_{p}\right) v_{k_{p}}^{2}\right]$ as in (4.7), we compute the quantities

$$
\begin{aligned}
\delta_{u} I_{\ell}(v)= & \sum_{p=1}^{N} r_{p}\left(\cos \left(\varphi_{p}\right) J \partial_{x_{\ell}} v_{k_{p}}^{1}+\sin \left(\varphi_{p}\right) J \partial_{x_{\ell}} v_{k_{p}}^{2}\right) \\
= & \sum_{p=1}^{N} r_{p} \cos \left(\varphi_{p}\right) J \partial_{x_{\ell}}\left(\begin{array}{c}
c_{0} \cdot k_{p} \cos \left(k_{p} \cdot x\right) \\
\left(g+\sigma\left|k_{p}\right|^{2}\right) \sin \left(k_{p} \cdot x\right)
\end{array}\right) \\
& +r_{p} \sin \left(\varphi_{p}\right) J \partial_{x_{\ell}}\left(\begin{array}{c}
-c_{0} \cdot k_{p} \sin \left(k_{p} \cdot x\right) \\
\left(g+\sigma\left|k_{p}\right|^{2}\right) \cos \left(k_{p} \cdot x\right)
\end{array}\right)
\end{aligned}
$$

and

$$
\int_{T(\Gamma)}\left(\delta_{u} I_{\ell}(v), \delta_{u} I_{j}(v)\right) d x=\sum_{p=1}^{N} r_{p}^{2}\left(k_{p}^{j} k_{p}^{\ell}\right)|T(\Gamma)|\left(\left(c_{0} \cdot k_{p}\right)^{2}+\left(g+\sigma\left|k_{p}\right|^{2}\right)^{2}\right)
$$

Let us suppose that $a=\left(a_{1}, a_{2}\right)$ is not collinear with any wave vector in the set $K$, and that $(v, c)$ satisfies $I(v+w(v, c))=a$. Define the quantity

$$
\begin{aligned}
m(v, c) & =\frac{1}{|v|^{2}} \int_{T(\Gamma)}\left(q(v, c), \delta_{u} I_{j}(v+w(v, c))\right) d x \\
& =\frac{1}{|v|^{2}}\left(\int_{T(\Gamma)}-\sum_{\ell=1}^{2}\left(c-c_{0}\right)_{\ell}\left(\delta_{u} I_{\ell}(v), \delta_{u} I_{j}(v)\right) d x+O\left(|v|^{3}\right)\right),
\end{aligned}
$$

which maps $X_{1} \times \mathbb{R}^{2}$ to $\mathbb{R}^{2}$, and whose vanishing implies (4.2). To analyze the set $m(v, c)=0$, evaluate $m$ on a line $\left\{v=\rho e: \rho \in \mathbb{R},|e|_{X_{1}}=1\right\}$ through the origin, setting $m_{e}(\rho, c)=m(\rho e, c)$. Clearly $m_{e}\left(0, c_{0}\right)=0$, and taking the derivative with 
respect to $c$,

$$
\begin{aligned}
\partial_{c_{\ell}} m_{e, j}(\rho, c) & =-\left.\frac{1}{|v|^{2}} \int_{T(\Gamma)}\left(\delta_{u} I_{\ell}(v), \delta_{u} I_{j}(v)\right) d x\right|_{v=\rho e}+O(|\rho|) \\
& =-\int_{T(\Gamma)}\left(\delta_{u} I_{\ell}(e), \delta_{u} I_{j}(e)\right) d x+O(|\rho|) .
\end{aligned}
$$

If this Jacobian is invertible, the implicit function theorem implies the existence of a solution $c(\rho)$ of $m_{e}(\rho, c)=0$ in a sufficiently small neighborhood of the origin. From (4.16) we see that $\partial_{c_{\ell}} m_{e j}(0, c)$ is a sum with nonnegative coefficients of symmetric rank one matrices $k_{p} k_{p}^{T}$. As long as two linearly independent $k_{p}$ of the sum have their respective coefficients nonzero, it is invertible, and the statement of the lemma holds for sufficiently small $\varepsilon$. Let us suppose then that $\partial_{c} m_{e}(0, c)$ is not invertible, for purposes of contradiction. Then both

$$
\begin{aligned}
\partial_{c_{j}} m_{e, \ell}\left(0, c_{0}\right) & =-\sum_{s} C_{p_{s}}^{(1)} k_{p_{s}}^{j} k_{p_{s}}^{\ell}, \\
I_{j}(e) & =\sum_{s} C_{p_{s}}^{(2)} k_{p_{s}}^{j},
\end{aligned}
$$

where all $k_{p_{s}}$ are collinear, and where the coefficients are $C_{p_{s}}^{(1)}=\left(\left(c_{0} \cdot k_{p}\right)^{2}+(g+\right.$ $\left.\left.\sigma\left|k_{p}\right|^{2}\right)^{2}\right)|T(\Gamma)|, C_{p_{s}}^{(2)}=\left(\left(c_{0} \cdot k_{p}\right)\left(g+\sigma\left|k_{p}\right|^{2}\right)\right)|T(\Gamma)|$. However, for $v=\rho e$, we have $\left(a_{1}, a_{2}\right)=\left(I_{1}(v+w(v, c)), I_{2}(v+w(v, c))\right)=\rho^{2}\left(I_{1}(e), I_{2}(e)\right)+O\left(|\rho|^{4}\right)$ and this in turn is $\rho^{2} \sum_{s} C_{p_{s}}^{(2)}\left(k_{p_{s}}^{1}, k_{p_{s}}^{2}\right)+O\left(|\rho|^{4}\right)$. For sufficiently small $\rho$ this is incompatible with the hypothesis of noncollinearity of the lemma, and hence $\partial_{c} m_{e}\left(0, c_{0}\right)$ must be invertible.

Once a solution $c(v)$ of $m(v, c)=0$ is established for $v \neq 0$, another argument using the statement of the implicit function theorem implies that $c(v)$ is locally real analytic away from the origin. Indeed, for $\rho \neq 0$ and for $e \in S_{1}=\left\{v \in X_{i}:|v|=1\right\}$ such that $\left(I_{1}(e), I_{2}(e)\right) \nsim k_{p}$, the implicit function theorem implies that the solution $c(v)=C(\rho e)$ is real analytic in the parameter $e$. This concludes the proof of the lemma.

5. Analyticity of the Dirichlet-Neumann operator. The one remaining detail in our proof is the analyticity of the Dirichlet-Neumann operator. This was first established in the two dimensional setting by R. Coifman and Y. Meyer [4], and in the three dimensional setting by W. Craig, U. Schanz, and C. Sulem [7]. The result in general $n$ dimensions was proven by D. Nicholls [19], as a generalization of the method of W. Craig, U. Schanz, and C. Sulem. All of these authors proved the following result in differing numbers of dimensions.

THEOREM 5.1. Consider functions $\eta$ such that $|\eta|_{L^{\infty}}<h R_{0},|\eta|_{C^{1}}<R_{0}$, and $|\eta|_{C^{s+1}}<\infty$. There exists $R_{0}>0$ such that the Dirichlet-Neumann operator $G(\eta)$ is analytic in $\eta$ in the neighborhood,

$$
\left\{\left.\eta|| \eta\right|_{C^{1}}<R_{0},|\eta|_{C^{s+1}}<\infty\right\},
$$

as a linear map in $\xi$ from $W^{s+1, q} \rightarrow W^{s, q}$.

In this theorem $C^{s}$ is the space of $s$ times continuously differentiable functions, and $W^{s, q}$ is the $L^{q}$ based Sobolev space of $s$ times differentiable functions. In his thesis D. Nicholls [19] modified this theorem in two dimensions by only requiring $\eta \in W^{s+1, q}$, which is used to prove the result of this paper in two dimensions with 
$\sigma=0$. The approach of W. Craig, U. Schanz, and C. Sulem [7] was to perform estimates on the Dirichlet-Neumann operator by always placing $C^{s}$ norms on $\eta$ terms and $W^{s, q}$ norms on $\xi$ terms. This produces a very clean result, which is not, however, useful for the applications in the present paper. In this section we replace the $C^{s+1}$ derivative with a $W^{s+1, q}$ at the cost of a $C^{2}$ derivative on $\xi$. In this section we generalize this procedure to higher dimensions for use in the three dimensional result.

5.1. An exact implicit formula. The method of W. Craig, U. Schanz, and C. Sulem [7] begins with an exact implicit formula for the Dirichlet-Neumann operator. The formula is in terms of smoothing and singular integral operators which one can analyze using the theorem of M. Christ and J. Journé [3]. In this section we consider $n$ dimensional real space $\mathbb{R}^{n}$ and will use the notation $x=\left(x^{\prime}, x_{n}\right) \in \mathbb{R}^{n-1} \times \mathbb{R}$ for a point in $\mathbb{R}^{n}$ rather than the $(x, y)$ notation of previous sections.

THEOREM 5.2. An exact implicit formula for the Dirichlet-Neumann operator in $n \geq 2$ dimensions is

$$
(I-B(\eta)) G(\eta) \xi=\left|D_{x^{\prime}}\right| \tanh \left(h\left|D_{x^{\prime}}\right|\right) \xi+A(\eta) \xi,
$$

where

$$
\begin{aligned}
& A(\eta) \zeta=-\left(1+e^{-2 h\left|D_{x^{\prime}}\right|}\right)^{-1}\left|D_{x^{\prime}}\right| M(\eta) \zeta, \\
& B(\eta) \zeta=-\left(1+e^{-2 h\left|D_{x^{\prime}}\right|}\right)^{-1}\left|D_{x^{\prime}}\right| L(\eta) \zeta
\end{aligned}
$$

and

$$
\begin{aligned}
M(\eta) \zeta & =\int m_{n}\left(x^{\prime}, y^{\prime}\right) \zeta\left(y^{\prime}\right) d y^{\prime} \\
L(\eta) \zeta & =\int l_{n}\left(x^{\prime}, y^{\prime}\right) \zeta\left(y^{\prime}\right) d y^{\prime}
\end{aligned}
$$

In two dimensions

$$
\begin{aligned}
m_{2}\left(x^{\prime}, y^{\prime}\right)= & \frac{1}{\pi}\left[\frac{1}{\left(x^{\prime}-y^{\prime}\right)^{2}}\left\{\frac{\left(x^{\prime}-y^{\prime}\right) \partial_{y^{\prime}} \eta\left(y^{\prime}\right)-\left(\eta\left(x^{\prime}\right)-\eta\left(y^{\prime}\right)\right)}{1+q_{1}^{2}}\right\}\right. \\
& +\frac{1}{\left(x^{\prime}-y^{\prime}\right)^{2}+4 h^{2}}\left\{\frac{\left(x^{\prime}-y^{\prime}\right) \partial_{y^{\prime}} \eta\left(y^{\prime}\right)+\left(\eta\left(x^{\prime}\right)+\eta\left(y^{\prime}\right)\right)}{1+\frac{4 h}{\sqrt{\left(x^{\prime}-y^{\prime}\right)^{2}+4 h^{2}}} q_{2}+q_{2}^{2}}\right\} \\
& \left.+\frac{2 h}{\left(x^{\prime}-y^{\prime}\right)^{2}+4 h^{2}}\left\{\frac{1}{1+\frac{4 h}{\sqrt{\left(x^{\prime}-y^{\prime}\right)^{2}+4 h^{2}}} q_{2}+q_{2}^{2}}-1\right\}\right]
\end{aligned}
$$

and

$$
l_{2}\left(x^{\prime}, y^{\prime}\right)=-\frac{1}{2 \pi}\left[\log \left(1+q_{1}^{2}\right)+\log \left(1+\frac{4 h}{\sqrt{\left(x^{\prime}-y^{\prime}\right)^{2}+4 h^{2}}} q_{2}+q_{2}^{2}\right)\right] .
$$

In these formulae we set

$$
\begin{aligned}
& q_{1}\left(x^{\prime}, y^{\prime}\right)=\frac{\eta\left(x^{\prime}\right)-\eta\left(y^{\prime}\right)}{\left|x^{\prime}-y^{\prime}\right|}, \\
& q_{2}\left(x^{\prime}, y^{\prime}\right)=\frac{\eta\left(x^{\prime}\right)+\eta\left(y^{\prime}\right)}{\sqrt{\left|x^{\prime}-y^{\prime}\right|^{2}+4 h^{2}}} .
\end{aligned}
$$


In $n \geq 3$ dimensions,

$$
\begin{aligned}
m_{n}\left(x^{\prime}, y^{\prime}\right)= & \frac{2}{\omega_{n}}\left[\frac{1}{\left|x^{\prime}-y^{\prime}\right|^{n}}\left\{\frac{\left(x^{\prime}-y^{\prime}\right) \cdot \nabla_{y^{\prime}} \eta\left(y^{\prime}\right)-\left(\eta\left(x^{\prime}\right)-\eta\left(y^{\prime}\right)\right)}{\left(1+q_{1}^{2}\right)^{n / 2}}\right\}\right. \\
& +\frac{1}{\left(\left|x^{\prime}-y^{\prime}\right|^{2}+4 h^{2}\right)^{\frac{n}{2}}}\left\{\frac{\left(x^{\prime}-y^{\prime}\right) \cdot \nabla_{y^{\prime}} \eta\left(y^{\prime}\right)+\left(\eta\left(x^{\prime}\right)+\eta\left(y^{\prime}\right)\right)}{\left(1+\frac{4 h}{\sqrt{\left|x^{\prime}-y^{\prime}\right|^{2}+4 h^{2}}} q_{2}+q_{2}^{2}\right)^{n / 2}}\right\} \\
& \left.+\frac{2 h}{\left(\left|x^{\prime}-y^{\prime}\right|^{2}+4 h^{2}\right)^{\frac{n}{2}}}\left\{\frac{1}{\left(1+\frac{4 h}{\sqrt{\left|x^{\prime}-y^{\prime}\right|^{2}+4 h^{2}}} q_{2}+q_{2}^{2}\right)^{n / 2}}-1\right\}\right]
\end{aligned}
$$

and

$$
\begin{aligned}
l_{n}\left(x^{\prime}, y^{\prime}\right) & =\frac{2}{(n-2) \omega_{n}}\left[\frac { 1 } { | x ^ { \prime } - y ^ { \prime } | ^ { n - 2 } } \left\{\frac{1}{\left.\left(1+q_{1}^{2}\right)^{(n-2) / 2}-1\right\}}\right.\right. \\
& \left.+\frac{1}{\left(\left|x^{\prime}-y^{\prime}\right|^{2}+4 h^{2}\right)^{(n-2) / 2}}\left\{\frac{1}{\left(1+\frac{4 h}{\sqrt{\left|x^{\prime}-y^{\prime}\right|^{2}+4 h^{2}}} q_{2}+q_{2}^{2}\right)^{(n-2) / 2}}-1\right\}\right],
\end{aligned}
$$

where $\omega_{n}=2 \sqrt{\pi}^{n} / \Gamma\left(\frac{n}{2}\right)$.

Proof. The proof of this result can be found in the paper of W. Craig, U. Schanz, and C. Sulem [7] for the case of three dimensions, or in the thesis of D. Nicholls [19] for the general case. The essence of the proof is that one can express solutions of Laplace's equation at a point in terms of the function itself and the fundamental solution. From here one takes appropriate derivatives, evaluates at the free surface, identifies the Dirichlet-Neumann operator, and then recognizes the linear terms as convolutions and writes them as $\left|D_{x^{\prime}}\right| \tanh \left(h\left|D_{x^{\prime}}\right|\right)$. In case that the depth $h$ is infinite, the above Fourier multiplier is replaced by $\left|D_{x^{\prime}}\right|$, the expression $q_{2}$ vanishes, and the subsequent analysis is similar but simpler. We will carry out the analysis for the case $h$ finite in the remainder of this section.

5.2. Proof of analyticity. The principal ingredients are the formulae of section 5.1 for the Dirichlet-Neumann operator and the theorems on singular and smoothing integral operators of section 5.3. The method we use is similar to the one employed by W. Craig, U. Schanz, and C. Sulem [7] and D. Nicholls [19]. We modify the approach by considering function spaces which are relevant to our current purpose. In particular, we will require only that $\eta \in W^{s+1, q}$ rather than $C^{s+1}$ in the two dimensional setting, and $\eta \in W^{s+2, q}$ rather than $C^{s+1}$ in the three dimensional setting, representing an improvement in the smoothness required of $\eta$-however, at the cost of the information and elegance of the proof of W. Craig, U. Schanz, and C. Sulem [7], in particular information involving $|\eta|_{C^{1}}$. 
By investigating the analyticity properties of the operators $A(\eta)$ and $B(\eta)$, along with the analyticity of the operator $(I-B(\eta))$, we will show that the DirichletNeumann operator itself is analytic. We begin by considering the operator $B(\eta)$.

THEOREM 5.3. In the setting of the two dimensional water wave problem $(n=2)$, if $1<q<\infty$ and $s>\max \left(\frac{1}{q}, 2\right)$, then $B(\eta)$ satisfies the estimate

$$
\|B(\eta) \xi\|_{W^{s, q}}<C\|\eta\|_{W^{s+1, q}}^{2}\|\xi\|_{W^{s, q}} .
$$

Furthermore, the operator $B(\eta)$ is analytic as a function of $\eta$ on the space $W^{s, q}$. In the setting of the three dimensional water wave problem $(n=3)$, if $1<q<\infty$ and $s>\max \left(\frac{2}{q}, 3\right)$, then $B(\eta)$ satisfies the estimate

$$
\|B(\eta) \xi\|_{W^{s, q}}<C\|\eta\|_{W^{s+2, q}}^{2}\|\xi\|_{W^{s, q}} .
$$

Furthermore, the operator $B(\eta)$ is analytic as a function of $\eta$ on the space $W^{s, q}$.

The proof of this theorem depends upon the following two lemmas which are proven in W. Craig, U. Schanz, and C. Sulem [7] and D. Nicholls [19].

Lemma 5.4. The operator $\left(1+e^{-2 h\left|D_{x^{\prime}}\right|}\right)^{-1}$ and the Riesz potential $R_{j}\left(D_{x^{\prime}}\right)=$ $i \frac{D_{x_{j}^{\prime}}}{\left|D_{x^{\prime}}\right|}$ are bounded on $W^{s, q}$ for $1<q<\infty$ and $s \geq 0$.

Lemma 5.5. Given functions $f \in C^{s}$ and $g \in W^{s, q}$ the following interpolation identity holds for some constant $K(s)$ :

$$
\|f g\|_{W^{s, q}} \leq K(s)\left[|f|_{L^{\infty}}\|g\|_{W^{s, q}}+|f|_{C^{s}}\|g\|_{L^{q}}\right] .
$$

We may now proceed with the proof of Theorem 5.3. Since

$$
\left|D_{x^{\prime}}\right|=-\sum_{j=1}^{n-1} R_{j}\left(D_{x^{\prime}}\right) \partial_{x_{j}^{\prime}}
$$

and we have Lemma 5.4, we need only consider two types of integral operators, $\partial_{x_{j}^{\prime}}$ applied to

$$
\begin{aligned}
P_{2} & =\int-\frac{1}{2 \pi} \log \left(1+q_{1}^{2}\right) \xi\left(y^{\prime}\right) d y^{\prime} \\
Q_{2} & =\int-\frac{1}{2 \pi} \log \left(1+\kappa_{h} q_{2}+q_{2}^{2}\right) \xi\left(y^{\prime}\right) d y^{\prime}
\end{aligned}
$$

in two dimensions and

$$
\begin{aligned}
P_{n} & =\int \frac{2}{(n-2) \omega_{n}} \frac{1}{\left|x^{\prime}-y^{\prime}\right|^{n-2}}\left\{\frac{1}{\left(1+q_{1}^{2}\right)^{(n-2) / 2}}-1\right\} \xi\left(y^{\prime}\right) d y^{\prime} \\
Q_{n} & =\int \frac{2}{(n-2) \omega_{n}} \frac{1}{\left(\left|x^{\prime}-y^{\prime}\right|^{2}+4 h^{2}\right)^{(n-2) / 2}} \\
& \times\left\{\frac{1}{\left(1+\kappa_{h} q_{2}+q_{2}^{2}\right)^{(n-2) / 2}}-1\right\} \xi\left(y^{\prime}\right) d y^{\prime}
\end{aligned}
$$

in $n \geq 3$ dimensions, where $\kappa_{h}=4 h / \sqrt{\left|x^{\prime}-y^{\prime}\right|^{2}+4 h^{2}}$. Applying the derivative operator to the above quantities results in the following formulae:

$$
\partial_{x^{\prime}} P_{2}=-\frac{1}{\pi} \int \frac{q_{1}}{1+q_{1}^{2}}\left[\frac{\partial_{x^{\prime}} \eta\left(x^{\prime}\right)}{\left|x^{\prime}-y^{\prime}\right|}-\frac{\left(\eta\left(x^{\prime}\right)-\eta\left(y^{\prime}\right)\right)\left(x^{\prime}-y^{\prime}\right)}{\left|x^{\prime}-y^{\prime}\right|^{3}}\right] \xi\left(y^{\prime}\right) d y^{\prime},
$$




$$
\begin{aligned}
\partial_{x^{\prime}} Q_{2}= & -\frac{1}{2 \pi} \int \frac{1}{1+\kappa_{h} q_{2}+q_{2}^{2}}\left[-q_{2} \frac{4 h\left(x^{\prime}-y^{\prime}\right)}{\left(\left|x^{\prime}-y^{\prime}\right|^{2}+4 h^{2}\right)^{3 / 2}}\right. \\
& \left.+\left(\kappa_{h}+2 q_{2}\right)\left\{\frac{\partial_{x^{\prime}} \eta\left(x^{\prime}\right)}{\left(\left|x^{\prime}-y^{\prime}\right|^{2}+4 h^{2}\right)^{1 / 2}}-\frac{\left(\eta\left(x^{\prime}\right)+\eta\left(y^{\prime}\right)\right)\left(x^{\prime}-y^{\prime}\right)}{\left(\left|x^{\prime}-y^{\prime}\right|^{2}+4 h^{2}\right)^{3 / 2}}\right\}\right] \xi\left(y^{\prime}\right) d y^{\prime}
\end{aligned}
$$

in two dimensions and

$$
\begin{aligned}
& \partial_{x_{j}^{\prime}} P_{n}=-\frac{2}{\omega_{n}} \int \frac{x_{j}^{\prime}-y_{j}^{\prime}}{\left|x^{\prime}-y^{\prime}\right|^{n}}\left\{\frac{1}{\left(1+q_{1}^{2}\right)^{(n-2) / 2}}-1\right\} \xi\left(y^{\prime}\right) d y^{\prime} \\
& -\frac{2}{\omega_{n}} \int \frac{1}{\left|x^{\prime}-y^{\prime}\right|^{n-2}} \frac{q_{1}}{\left(1+q_{1}^{2}\right)^{n / 2}}\left[\frac{\partial_{x_{j}^{\prime}} \eta\left(x^{\prime}\right)}{\left|x^{\prime}-y^{\prime}\right|}\right. \\
& \left.-\frac{\left(\eta\left(x^{\prime}\right)-\eta\left(y^{\prime}\right)\right)\left(x_{j}^{\prime}-y_{j}^{\prime}\right)}{\left|x^{\prime}-y^{\prime}\right|^{3}}\right] \xi\left(y^{\prime}\right) d y^{\prime}, \\
& \partial_{x_{j}^{\prime}} Q_{n}=\frac{2}{\omega_{n}} \int \frac{x_{j}^{\prime}-y_{j}^{\prime}}{\left(\left|x^{\prime}-y^{\prime}\right|^{2}+4 h^{2}\right)^{n / 2}}\left\{\frac{1}{\left(1+\kappa_{h} q_{2}+q_{2}^{2}\right)^{(n-2) / 2}}-1\right\} \xi\left(y^{\prime}\right) d y^{\prime} \\
& +\frac{2}{(n-2) \omega_{n}} \int \frac{1}{\left(\left|x^{\prime}-y^{\prime}\right|^{2}+4 h^{2}\right)^{(n-2) / 2}} \frac{1}{\left(1+\kappa_{h} q_{2}+q_{2}^{2}\right)^{n / 2}} \\
& \times\left\{-q_{2} \frac{4 h\left(x_{j}^{\prime}-y_{j}^{\prime}\right)}{\left(\left|x^{\prime}-y^{\prime}\right|^{2}+4 h^{2}\right)^{3 / 2}}\right. \\
& \left.+\left(\kappa_{h}+2 q_{2}\right) \cdot\left[\frac{\partial_{x_{j}^{\prime}} \eta\left(x^{\prime}\right)}{\left(\left|x^{\prime}-y^{\prime}\right|^{2}+4 h^{2}\right)^{1 / 2}}-\frac{\left(\eta\left(x^{\prime}\right)+\eta\left(y^{\prime}\right)\right)\left(x_{k}^{\prime}-y_{k}^{\prime}\right)}{\left(\left|x^{\prime}-y^{\prime}\right|^{2}+4 h^{2}\right)^{3 / 2}}\right]\right\} \xi\left(y^{\prime}\right) d y^{\prime}
\end{aligned}
$$

in $n \geq 3$ dimensions. It is not difficult to see that the singular and smoothing integral operator theorems of section 5.3 apply to the above operators if we keep in mind that $d=n-1$. Indeed, in the seven principal terms appearing in the expression for the operator $\partial_{x_{j}^{\prime}} Q_{n}$ and in the notation of Theorem 5.21, we have $(p, \rho, \lambda)=(1, n-2,1)$, $(2, n-2,0),(1, n, 0),(0, n-1,1),(1, n-1,0),(1, n, 0)$, and $(0, n, 1)$. It is also useful to use Lemma 5.5 to pull out functions which depend on $x^{\prime}$ from the $y^{\prime}$ integrals, and interpolate.

The goal is not to show that $B(\eta)$ is analytic but rather to show that $(I-B(\eta))^{-1}$ is analytic. With this in mind we make the following estimate concerning powers of the operator $B(\eta)$ and then conclude analyticity of $(I-B(\eta))^{-1}$.

COROLlaRY 5.6. In the setting of the two dimensional water wave problem $(n=$ 2 ), if $1<q<\infty$ and $s>\max \left(\frac{1}{q}, 2\right)$, then the powers of $B(\eta)$ satisfy the following estimate:

$$
\left\|B(\eta)^{j} \xi\right\|_{W^{s, q}}<C^{j}\|\eta\|_{W^{s+1, q}}^{2 j}\|\xi\|_{W^{s, q}} .
$$

Thus, for $\|\eta\|_{W^{s+1, q}}$ small enough, the operator $(I-B(\eta))^{-1}$ exists, satisfies the 
estimate

$$
\left\|(I-B(\eta))^{-1} \xi\right\|_{W^{s, q}}<1+\tilde{C}\|\xi\|_{W^{s, q}},
$$

and is analytic as a function of $\eta$ on $W^{s, q}$. In the setting of the three dimensional water wave problem $(n=3)$, if $1<q<\infty$ and $s>\max \left(\frac{2}{q}, 3\right)$, then the powers of $B(\eta)$ satisfy the following estimate:

$$
\left\|B(\eta)^{j} \xi\right\|_{W^{s, q}}<C^{j}\|\eta\|_{W^{s+2, q}}^{2 j}\|\xi\|_{W^{s, q}} .
$$

Thus, for $\|\eta\|_{W^{s+2, q}}$ small enough, the operator $(I-B(\eta))^{-1}$ exists, satisfies the estimate

$$
\left\|(I-B(\eta))^{-1} \xi\right\|_{W^{s, q}}<1+\tilde{C}\|\xi\|_{W^{s, q}},
$$

and is analytic as a function of $\eta$ on $W^{s, q}$.

Proof. We restrict to the case of $n=2$ as the $n=3$ case is virtually identical. We already have the estimate

$$
\|B(\eta) \xi\|_{W^{s, q}}<C\|\eta\|_{W^{s+1, q}}^{2}\|\xi\|_{W^{s, q}},
$$

and we now proceed via induction. The case $j=1$ is clearly true, so we assume that the corollary is true for $j$ and analyze $(j+1)$,

$$
\begin{aligned}
\left\|B(\eta)^{j+1} \xi\right\|_{W^{s, q}} & =\left\|B(\eta)^{j} B(\eta) \xi\right\|_{W^{s, q}} \\
& <C^{j}\|\eta\|_{W^{s+1, q}}^{2 j}\|B(\eta) \xi\|_{W^{s, q}} \\
& <C^{j+1}\|\eta\|_{W^{s+1, q}}^{2(j+1)}\|\xi\|_{W^{s, q}} .
\end{aligned}
$$

Therefore, the estimate on the $j$ th power holds true. To compute $(I-B(\eta))^{-1}$ we use the Neumann series

$$
(I-B(\eta))^{-1}=I+\sum_{j=1}^{\infty} B^{j}(\eta)
$$

and note that it converges in the radius of convergence of the series

$$
\sum_{j=1}^{\infty} C^{j}\|\eta\|_{W^{s+1, q}}^{2 j} .
$$

In other words, the Neumann series converges when $\|\eta\|_{W^{s+1, q}}<1 / \sqrt{C}$, and we are done.

We now turn our attention to the operators $A(\eta)$. While the operators $B(\eta)$ map $W^{s, q}$ into $W^{s, q}$ and thereby preserve derivatives, we should expect the operators $A(\eta)$ to map $W^{s+1, q}$ into $W^{s, q}$. This is incorporated into our proof by using a variation of integration by parts, given below, to remove all $\partial_{y^{\prime}}$ derivatives from $\eta\left(y^{\prime}\right)$ terms and place them elsewhere. We give the version of integration by parts in the following lemma proven in W. Craig, U. Schanz, and C. Sulem [7] and D. Nicholls [19].

Lemma 5.7. Consider $x, y \in \mathbb{R}^{n}, R\left(q_{1}\right)$ an odd continuous function of $q_{1}$, and $\eta \in C^{1}\left(\mathbb{R}^{n}\right)$; then we have that

$$
\int \frac{x-y}{|x-y|^{n}} \cdot \nabla_{y}\left(R\left(q_{1}\right)\right) \xi(y) d y=-\int R\left(q_{1}\right) \frac{x-y}{|x-y|^{n}} \cdot \nabla_{y} \xi(y) d y .
$$


We are can now prove an estimate on the operator $A(\eta)$ which is given in the following theorem.

THEOREM 5.8. In the setting of the two dimensional water wave problem $(n=2)$, if $1<q<\infty$ and $s>\max \left(\frac{1}{q}, 2\right)$, then $A(\eta)$ satisfies the estimate

$$
\|A(\eta) \xi\|_{W^{s, q}}<C\|\eta\|_{W^{s+1, q}}^{2}\|\xi\|_{W^{s+1, q}} .
$$

Furthermore, the operator $A(\eta)$ is analytic as a function of $\eta$ from the space $W^{s+1, q}$ to the space $W^{s, q}$. In the setting of the three dimensional water wave problem $(n=3)$, if $1<q<\infty$ and $s>\max \left(\frac{2}{q}, 3\right)$, then $A(\eta)$ satisfies the estimate

$$
\|A(\eta) \xi\|_{W^{s, q}}<C\|\eta\|_{W^{s+2, q}}^{2}\|\xi\|_{W^{s+1, q}} .
$$

Furthermore, the operator $A(\eta)$ is analytic as a function of $\eta$ from the space $W^{s+1, q}$ to the space $W^{s, q}$.

Proof. As before, since

$$
\left|D_{x^{\prime}}\right|=-\sum_{j=1}^{n-1} R_{j}\left(D_{x^{\prime}}\right) \partial_{x_{j}^{\prime}}
$$

is the Riesz potential given in Lemma 5.4, we need consider only two types of integral operators, $\partial_{x_{j}^{\prime}}$ applied to

$$
\begin{aligned}
P_{2}= & \int \frac{1}{\pi} \frac{1}{\left(x^{\prime}-y^{\prime}\right)^{2}}\left\{\frac{\left(x^{\prime}-y^{\prime}\right) \partial_{y^{\prime}} \eta\left(y^{\prime}\right)-\left(\eta\left(x^{\prime}\right)-\eta\left(y^{\prime}\right)\right)}{1+q_{1}^{2}}\right\} \xi\left(y^{\prime}\right) d y^{\prime}, \\
Q_{2}= & \int \frac{1}{\pi} \frac{1}{\left(x^{\prime}-y^{\prime}\right)^{2}+4 h^{2}}\left\{\frac{\left(x^{\prime}-y^{\prime}\right) \partial_{y^{\prime}} \eta\left(y^{\prime}\right)}{1+\kappa_{h} q_{2}+q_{2}^{2}}\right. \\
& \left.+\frac{\left(\eta\left(x^{\prime}\right)+\eta\left(y^{\prime}\right)\right)}{1+\kappa_{h} q_{2}+q_{2}^{2}}\right\} \xi\left(y^{\prime}\right) d y^{\prime}, \\
R_{2}= & \int \frac{1}{\pi} \frac{2 h}{\left(x^{\prime}-y^{\prime}\right)^{2}+4 h^{2}}\left\{\frac{1}{1+\kappa_{h} q_{2}+q_{2}^{2}}-1\right\} \xi\left(y^{\prime}\right) d y^{\prime}
\end{aligned}
$$

in two dimensions and

$$
\begin{aligned}
P_{n}= & \int \frac{2}{\omega_{n}} \frac{1}{\left|x^{\prime}-y^{\prime}\right|^{n}}\left\{\frac{\left(x^{\prime}-y^{\prime}\right) \cdot \nabla_{y^{\prime}} \eta\left(y^{\prime}\right)-\left(\eta\left(x^{\prime}\right)-\eta\left(y^{\prime}\right)\right)}{\left(1+q_{1}^{2}\right)^{n / 2}}\right\} \xi\left(y^{\prime}\right) d y^{\prime}, \\
Q_{n}= & \int \frac{2}{\omega_{n}} \frac{1}{\left(\left|x^{\prime}-y^{\prime}\right|^{2}+4 h^{2}\right)^{n / 2}}\left\{\frac{\left(x^{\prime}-y^{\prime}\right) \cdot \nabla_{y^{\prime}} \eta\left(y^{\prime}\right)}{\left(1+\kappa_{h} q_{2}+q_{2}^{2}\right)^{n / 2}}\right. \\
& \left.+\frac{\left(\eta\left(x^{\prime}\right)+\eta\left(y^{\prime}\right)\right)}{\left(1+\kappa_{h} q_{2}+q_{2}^{2}\right)^{n / 2}}\right\} \xi\left(y^{\prime}\right) d y^{\prime}, \\
R_{n}= & \int \frac{2}{\omega_{n}} \frac{2 h}{\left(\left|x^{\prime}-y^{\prime}\right|^{2}+4 h^{2}\right)^{n / 2}}\left\{\frac{1}{\left(1+\kappa_{h} q_{2}+q_{2}^{2}\right)^{n / 2}}-1\right\} \xi\left(y^{\prime}\right) d y^{\prime}
\end{aligned}
$$

in $n \geq 3$ dimensions. All we need to do is apply the differential operator $\partial_{x_{j}^{\prime}}$ to each of these integrals, use Lemma 5.7 wherever appropriate, and then note that the singular 
and smoothing integral operator theorems of section 5.3 can be used. Keeping in mind the facts that

$$
\begin{aligned}
\partial_{x_{j}^{\prime}} q_{1}\left(x^{\prime}, y^{\prime}\right) & =\frac{\partial_{x_{j}^{\prime}} \eta\left(x^{\prime}\right)}{\left|x^{\prime}-y^{\prime}\right|}-\frac{\left(\eta\left(x^{\prime}\right)-\eta\left(y^{\prime}\right)\right)\left(x_{j}^{\prime}-y_{j}^{\prime}\right)}{\left|x^{\prime}-y^{\prime}\right|^{3}} \\
\partial_{x_{j}^{\prime}} q_{2}\left(x^{\prime}, y^{\prime}\right) & =\frac{\partial_{x_{j}^{\prime}} \eta\left(x^{\prime}\right)}{\left(\left|x^{\prime}-y^{\prime}\right|^{2}+4 h^{2}\right)^{1 / 2}}-\frac{\left(\eta\left(x^{\prime}\right)+\eta\left(y^{\prime}\right)\right)\left(x_{j}^{\prime}-y_{j}^{\prime}\right)}{\left(\left|x^{\prime}-y^{\prime}\right|^{2}+4 h^{2}\right)^{3 / 2}} \\
\partial_{x_{j}^{\prime}} \kappa_{h}\left(x^{\prime}, y^{\prime}\right) & =-\frac{4 h\left(x_{j}^{\prime}-y_{j}^{\prime}\right)}{\left(\left|x^{\prime}-y^{\prime}\right|^{2}+4 h^{2}\right)^{3 / 2}}
\end{aligned}
$$

we compute the derivatives in two dimensions as

$$
\begin{aligned}
\partial_{x^{\prime}} P_{2}= & -\frac{2}{\pi} \int \frac{1}{\left(x^{\prime}-y^{\prime}\right)^{3}}\left\{\frac{\left(x^{\prime}-y^{\prime}\right) \cdot \nabla_{y^{\prime}} \eta\left(y^{\prime}\right)-\left(\eta\left(x^{\prime}\right)-\eta\left(y^{\prime}\right)\right)}{\left(1+q_{1}^{2}\right)^{n / 2}}\right\} \xi\left(y^{\prime}\right) d y^{\prime} \\
& +\frac{1}{\pi} \int \frac{1}{\left(x^{\prime}-y^{\prime}\right)^{2}}\left\{\frac{\partial_{y^{\prime}} \eta\left(y^{\prime}\right)-\partial_{x^{\prime}} \eta\left(x^{\prime}\right)}{1+q_{1}^{2}}\right. \\
& \left.-\frac{\left[\left(x^{\prime}-y^{\prime}\right) \partial_{y^{\prime}} \eta\left(y^{\prime}\right)-\left(\eta\left(x^{\prime}\right)-\eta\left(y^{\prime}\right)\right)\right] 2 q_{1} \partial_{x^{\prime}} q_{1}}{\left(1+q_{1}^{2}\right)^{2}}\right\} \xi\left(y^{\prime}\right) d y^{\prime}, \\
\partial_{x^{\prime}} Q_{2}= & -\frac{2}{\pi} \int \frac{x^{\prime}-y^{\prime}}{\left(\left(x^{\prime}-y^{\prime}\right)^{2}+4 h^{2}\right)^{2}}\left\{\frac{\left(x^{\prime}-y^{\prime}\right) \partial_{y^{\prime}} \eta\left(y^{\prime}\right)+\left(\eta\left(x^{\prime}\right)+\eta\left(y^{\prime}\right)\right)}{1+\kappa_{h} q_{2}+q_{2}^{2}}\right\} \xi\left(y^{\prime}\right) d y^{\prime} \\
& +\frac{1}{\pi} \int \frac{1}{\left(x^{\prime}-y^{\prime}\right)^{2}+4 h^{2}}\left\{\frac{\partial_{y^{\prime}} \eta\left(y^{\prime}\right)+\partial_{x^{\prime}} \eta\left(x^{\prime}\right)}{1+\kappa_{h} q_{2}+q_{2}^{2}}\right. \\
& \left.-\frac{\left[\left(x^{\prime}-y^{\prime}\right) \partial_{y^{\prime}} \eta\left(y^{\prime}\right)+\left(\eta\left(x^{\prime}\right)+\eta\left(y^{\prime}\right)\right)\right]\left(q_{2} \partial_{x^{\prime}} \kappa_{h}+\left(\kappa_{h}+2 q_{2}\right) \partial_{x^{\prime}} q_{2}\right)}{\left(1+\kappa_{h} q_{2}+q_{2}^{2}\right)^{2}}\right\} \xi\left(y^{\prime}\right) d y^{\prime}, \\
\partial_{x^{\prime}} R_{2}= & -\frac{2}{\pi} \int \frac{2 h\left(x^{\prime}-y^{\prime}\right)}{\left(\left(x^{\prime}-y^{\prime}\right)^{2}+4 h^{2}\right)^{2}}\left\{\frac{1}{1+\kappa_{h} q_{2}+q_{2}^{2}}-1\right\} \xi\left(y^{\prime}\right) d y^{\prime} \\
& -\frac{1}{\pi} \int \frac{2 h}{\left(x^{\prime}-y^{\prime}\right)^{2}+4 h^{2}} \frac{\left(q_{2} \partial_{x^{\prime}} \kappa_{h}+\left(\kappa_{h}+2 q_{2}\right) \partial_{x^{\prime}} q_{2}\right)}{\left(1+\kappa_{h} q_{2}+q_{2}^{2}\right)^{2}}
\end{aligned}
$$

and in $n=3$ dimensions as

$$
\begin{aligned}
\partial_{x_{j}^{\prime}} P_{n}= & \frac{2}{\omega_{n}} \int \frac{x_{j}^{\prime}-y_{j}^{\prime}}{\left|x^{\prime}-y^{\prime}\right|^{n+2}}\left\{\frac{\left(x^{\prime}-y^{\prime}\right) \cdot \nabla_{y^{\prime}} \eta\left(y^{\prime}\right)-\left(\eta\left(x^{\prime}\right)-\eta\left(y^{\prime}\right)\right)}{\left(1+q_{1}^{2}\right)^{n / 2}}\right\} \xi\left(y^{\prime}\right) d y^{\prime} \\
& +\frac{2}{\omega_{n}} \int \frac{1}{\left|x^{\prime}-y^{\prime}\right|^{n}}\left\{\frac{\partial_{y_{j}^{\prime}} \eta\left(y^{\prime}\right)-\partial_{x_{j}^{\prime}} \eta\left(x^{\prime}\right)}{\left(1+q_{1}^{2}\right)^{n / 2}}\right. \\
& \left.-\frac{\left[\left(x^{\prime}-y^{\prime}\right) \cdot \nabla_{y^{\prime}} \eta\left(y^{\prime}\right)-\left(\eta\left(x^{\prime}\right)-\eta\left(y^{\prime}\right)\right)\right]\left(n q_{1} \partial_{x_{j}^{\prime}} q_{1}\right)}{\left(1+q_{1}^{2}\right)^{n}}\right\} \xi\left(y^{\prime}\right) d y^{\prime}, \\
\partial_{x_{j}^{\prime}} Q_{n}= & -\frac{2 n}{\omega_{n}} \int \frac{x_{j}^{\prime}-y_{j}^{\prime}}{\left(\left|x^{\prime}-y^{\prime}\right|^{2}+4 h^{2}\right)^{(n+2) / 2}} \\
& \times\left\{\frac{\left(x^{\prime}-y^{\prime}\right) \cdot \nabla_{y^{\prime}} \eta\left(y^{\prime}\right)+\left(\eta\left(x^{\prime}\right)+\eta\left(y^{\prime}\right)\right)}{\left(1+\kappa_{h} q_{2}+q_{2}^{2}\right)^{n / 2}}\right\} \xi\left(y^{\prime}\right) d y^{\prime}
\end{aligned}
$$




$$
\begin{aligned}
+ & \frac{2}{\omega_{n}} \int \frac{1}{\left(\left|x^{\prime}-y^{\prime}\right|^{2}+4 h^{2}\right)^{n / 2}}\left\{\frac{\partial_{y_{j}^{\prime}} \eta\left(y^{\prime}\right)+\partial_{x_{j}^{\prime}} \eta\left(x^{\prime}\right)}{\left(1+\kappa_{h} q_{2}+q_{2}^{2}\right)^{n / 2}}\right. \\
& \left.-\frac{\left[\left(x^{\prime}-y^{\prime}\right) \cdot \nabla_{y^{\prime}} \eta\left(y^{\prime}\right)+\left(\eta\left(x^{\prime}\right)+\eta\left(y^{\prime}\right)\right)\right]\left(q_{2} \partial_{x_{j}^{\prime}} \kappa_{h}+\left(\kappa_{h}+2 q_{2}\right) \partial_{x_{j}^{\prime}} q_{2}\right)}{\left(1+\kappa_{h} q_{2}+q_{2}^{2}\right)^{n}}\right\} \xi\left(y^{\prime}\right) d y^{\prime}, \\
\partial_{x_{j}^{\prime}} R_{n}= & -\frac{2 n}{\omega_{n}} \int \frac{2 h\left(x_{j}^{\prime}-y_{j}^{\prime}\right)}{\left(\left|x^{\prime}-y^{\prime}\right|^{2}+4 h^{2}\right)^{(n+2) / 2}}\left\{\frac{1}{\left(1+\kappa_{h} q_{2}+q_{2}^{2}\right)^{n / 2}}-1\right\} \xi\left(y^{\prime}\right) d y^{\prime} \\
& -\frac{n}{\omega_{n}} \int \frac{2 h}{\left(\left|x^{\prime}-y^{\prime}\right|^{2}+4 h^{2}\right)^{n / 2}} \frac{q_{2} \partial_{x_{j}^{\prime}} \kappa_{h}+\left(\kappa_{h}+2 q_{2}\right) \partial_{x_{j}^{\prime}} q_{2}}{\left(1+\kappa_{h} q_{2}+q_{2}^{2}\right)^{(n+2) / 2}} \xi\left(y^{\prime}\right) d y^{\prime} .
\end{aligned}
$$

As noted before, we can now use the theorems of section 5.3 in combination with Lemma 5.7 to arrive at the conclusion of the theorem.

Now that we have Corollary 5.6 and Theorem 5.8, we can finally state and prove the theorem regarding analyticity of the Dirichlet-Neumann operator.

THEOREM 5.9. In the setting of the two dimensional water wave problem $(n=2)$, if $1<q<\infty$ and $s>\max \left(\frac{1}{q}, 2\right)$, then the Dirichlet-Neumann operator $G(\eta) \xi$ is analytic as a function of $\eta \in W^{s+1, q}$, as a bounded linear operator from $W^{s+1, q}$ to $W^{s, q}$. In the setting of the three dimensional water wave problem $(n=3)$, if $1<q<\infty$ and $s>\max \left(\frac{2}{q}, 3\right)$, then the Dirichlet-Neumann operator $G(\eta) \xi$ is analytic as a function of $\eta \in W^{s+2, q}$, as a bounded linear operator from $W^{s+1, q}$ to $W^{s, q}$.

Proof. Theorem 5.2 gives the exact implicit formula

$$
(I-B(\eta)) G(\eta) \xi=\left|D_{x^{\prime}}\right| \tanh \left(h\left|D_{x^{\prime}}\right|\right) \xi+A(\eta) \xi,
$$

and thus, since $(I-B(\eta))$ is boundedly invertible, we can write

$$
G(\eta) \xi=(I-B(\eta))^{-1}\left|D_{x^{\prime}}\right| \tanh \left(h\left|D_{x^{\prime}}\right|\right) \xi+(I-B(\eta))^{-1} A(\eta) \xi .
$$

Since Corollary 5.6 and Theorem 5.8 give us analyticity and appropriate boundedness of relevant operators we have the statement of the theorem.

5.3. Singular and smoothing integral operators. In this section we briefly outline the statement and proofs of theorems concerning the boundedness properties of certain singular and smoothing integral operators relevant to the proof the the analyticity of the Dirichlet-Neumann operator outlined above. The bulk of these theorems were first presented in W. Craig, U. Schanz, and C. Sulem [7] and U. Schanz $[25]$ in general $n$ dimensions for use in the analyticity proof of the Dirichlet-Neumann operator in three dimensions where $\eta \in C^{s+1}$ and $\xi \in W^{s, q}$. The others were first presented by D. Nicholls [19] in one dimension for use in the proof of the analyticity of the Dirichlet-Neumann operator in two dimensions which only required that $\eta, \xi \in$ $W^{s, q}$ for $s>\frac{d}{q}$. Here we extend the program of D. Nicholls [19] by proving his result in $d$ dimensions, and further reducing the smoothness required of $\eta$ and $\xi$.

Let $x, y \in \mathbb{R}^{d}$, and consider functions $\eta: \mathbb{R}^{d} \rightarrow \mathbb{R}$ and $\xi: \mathbb{R}^{d} \rightarrow \mathbb{R}$. Our goal is to study two classes of integral operators. The first is singular and has the general form

$$
C_{p}(\eta) \xi(x)=\int k(x-y) c_{p}\left(q_{1}\right) \xi(y) d y
$$


where $k(x-y)$ is a convolution kernel of Calderón-Zygmund type satisfying standard estimates outlined below. Recall that $q_{1}(\eta ; x, y)=(\eta(x)-\eta(y)) /|x-y|$, and consider $c_{p}: \mathbb{R} \rightarrow \mathbb{R}$ which is analytic in the interval $|z|<R_{0}$ such that $c_{p}(z)=\mathcal{O}\left(|z|^{p}\right)$ for $|z|$ small. The second class of operators is smoothing and has the general form

$$
C_{p, h}(\eta) \xi(x)=\int K_{h}(x-y) c_{p, h}\left(q_{2}, \kappa_{h}\right) \xi(y) d y
$$

where

$$
K_{h}(x)=\frac{1}{\left(|x|^{2}+4 h^{2}\right)^{\rho / 2}} \prod_{l=1}^{d}\left(\frac{x_{l}}{\left(|x|^{2}+4 h^{2}\right)^{1 / 2}}\right)^{\beta_{l}}
$$

with $\beta=\sum_{l=1}^{d} \beta_{l}$,

$$
q_{2}(\eta ; x, y)=\frac{\eta(x)+\eta(y)}{\left(|x-y|^{2}+4 h^{2}\right)^{1 / 2}}, \quad \kappa_{h}(x, y)=\frac{4 h}{\left(|x-y|^{2}+4 h^{2}\right)^{1 / 2}} .
$$

We consider $c_{p, h}(z, w): \mathbb{R}^{2} \rightarrow \mathbb{R}$ which is analytic for $\left\{|z|<R_{0},|w|<2\right\}$ such that $c_{p, h}(z, w)=\mathcal{O}\left(|z|^{p}|w|^{\lambda}\right)$ for $|z|$ and $|w|$ small. We will require that $p+\rho+\lambda>d$. We will establish Sobolev estimates for the operators $C_{p}$ and $C_{p, h}$ in the following theorems. The first, concerning bounds on $C_{p}$, requires the use of a deep theorem of M. Christ and J. Journé [3] on $L^{q}$ bounds for Calderón-Zygmund commutators. The theorem concerning bounds on the operators $C_{p, h}$ requires nothing more than careful estimates as the operator is smoothing rather than singular.

To place ourselves in the setting for the theorem of M. Christ and J. Journé [3], we define the standard estimates.

Definition 5.10. A kernel $K$ on $\mathbb{R}^{d}$ is said to satisfy standard estimates if there exist $\delta>0$ and $c_{K}<\infty$ such that for all distinct $x, y \in \mathbb{R}^{d}$ and all $z \in \mathbb{R}^{d}$ such that $|x-z| \leq \frac{|x-y|}{2}$,

(i) $|K(x, y)| \leq c_{K}|x-y|^{-d}$;

(ii) $|K(x, y)-K(z, y)| \leq c_{K}\left(\frac{|x-z|}{|x-y|}\right)^{\delta}|x-y|^{-d}$;

(iii) $|K(y, x)-K(y, z)| \leq c_{K}\left(\frac{|x-z|}{|x-y|}\right)^{\delta}|x-y|^{-d}$.

With this definition in hand we state the following theorem.

Theorem 5.11 (M. Christ and J. Journé [3, Theorem 4]). Consider the singular integral operator with kernel

$$
L(x-y) \prod_{j=1}^{m}\left(\int_{0}^{1} b_{j}(t x+(1-t) y) d t\right),
$$

where each $b_{j} \in L^{\infty}$ and $L$ satisfies the standard estimates. Then for $0<q<\infty$,

$$
\begin{array}{r}
\left\|\int L(x-y) \prod_{j=1}^{m}\left(\int_{0}^{1} b_{j}(t x+(1-t) y) d t\right) \xi(y) d y\right\|_{L^{q}} \\
\leq c_{q} c_{L} m^{N}\left(\prod_{j=1}^{m}\left|b_{j}\right|_{L^{\infty}}\right)\|\xi\|_{L^{q}} .
\end{array}
$$


One may take $N=2+\delta_{L}$, for $\delta_{L}$ and $c_{L}$ which appear in the standard estimates.

The first step in analyzing the boundedness of the operators $C_{p}(\eta)$ is to first consider the simplified operator

$$
S_{p}\left(\eta_{1}, \ldots, \eta_{p}\right) \xi(x)=\int K(x-y)\left(\prod_{j=1}^{p} q_{1}\left(\eta_{j}\right)\right) \xi(y) d y,
$$

where $K$ is a Calderón-Zygmund kernel which satisfies standard estimates. There are two related theorems which one can prove concerning this operator. The first is due to W. Craig, U. Schanz, and C. Sulem [7].

Theorem 5.12 (W. Craig, U. Schanz, and C. Sulem [7]). Let $\eta_{1}, \ldots, \eta_{p} \in C^{1}$, then the singular integral operator $S_{p}\left(\eta_{1}, \ldots, \eta_{p}\right)$ is bounded on $L^{q}$ and satisfies

$$
\left\|S_{p}\left(\eta_{1}, \ldots, \eta_{p}\right) \xi\right\|_{L^{q}} \leq C_{0} p^{M}\left(\prod_{j=1}^{p}\left|\eta_{j}\right|_{C^{1}}\right)\|\xi\|_{L^{q}}
$$

with exponent $M=3+\min \left(\delta_{K}, 1\right)$.

The second is an extension to general $d$ dimensions of a result proven by D. Nicholls [19].

TheOrEm 5.13. Let $1 \leq r \leq p, \eta_{j} \in C^{2}$ for $j \neq r, \xi \in C^{1} \cap W^{1, q}$, and $\eta_{r} \in L^{\infty} \cap L^{q}$. Consider $K$ of the form

$$
K(x, y)=\left\{\begin{array}{l}
\frac{1}{|x-y|^{d}}, \\
\frac{x-y}{|x-y|^{d+1}} .
\end{array}\right.
$$

Then the singular integral operator $S_{p}\left(\eta_{1}, \ldots, \eta_{p}\right)$ is bounded on $L^{q}$ and satisfies

$$
\begin{aligned}
\left\|S_{p}\left(\eta_{1}, \ldots, \eta_{p}\right) \xi\right\|_{L^{q}} \leq & C\left\{\left|\eta_{r}\right|_{L^{\infty}}\left(\prod_{j=1, j \neq r}^{p}\left|\eta_{j}\right|_{C^{1}}\right)\left\|\partial_{y} \xi\right\|_{L^{q}}\right. \\
& +\left|\eta_{r}\right|_{L^{\infty}} \sum_{s=1, s \neq r}^{p}\left(\prod_{j=1, j \neq r, s}^{p}\left|\eta_{j}\right|_{C^{1}}\right)\left|\eta_{s}\right|_{C^{2}}\|\xi\|_{L^{q}} \\
& +\left(\prod_{j=1, j \neq r}^{p}\left|\eta_{j}\right|_{C^{1}}\right)|\xi|_{C^{1}}\left\|\eta_{r}\right\|_{L^{q}} \\
& +|\xi|_{L^{\infty}}\left(\prod_{j=1, j \neq r}^{p}\left|\eta_{j}\right|_{C^{1}}\right)\left\|\partial_{y} \eta_{r}\right\|_{L^{q}} \\
& \left.+|\xi|_{L^{\infty}} \sum_{s=1, s \neq r}^{p}\left(\prod_{j=1, j \neq r, s}^{p}\left|\eta_{j}\right|_{C^{1}}\right)\left|\eta_{s}\right|_{C^{2}}\left\|\eta_{r}\right\|_{L^{q}}\right\}
\end{aligned}
$$

The proofs of both of these results rely on the following lemmas. We state without proof the first two (see [19]) which are used in the proof of Theorem 5.12.

Lemma 5.14. Suppose that $x \neq y$. If $f_{1}, \ldots, f_{p} \in C^{1}$, then

$$
\prod_{j=1}^{p} q_{1}\left(f_{j}\right)=\sum_{l \in \mathcal{L}}\left(\prod_{k=1}^{p} \frac{(x-y) l_{k}}{|x-y|}\right)\left(\int_{0}^{1} \partial_{x_{l_{k}}} f_{j}(t x+(1-t) y) d t\right),
$$


where $\mathcal{L}$ is the set of all integer $p$-tuples $\left(l_{1}, \ldots, l_{p}\right)$ such that $1 \leq l_{1}, \ldots, l_{p} \leq d$.

LEMma 5.15. If $k(x, y)$ is a Calderón-Zygmund kernel satisfying standard estimates, then the kernel of the form

$$
\zeta(x, y)=k(x, y) \prod_{k=1}^{p} \frac{(x-y)_{l_{k}}}{|x-y|}
$$

where $1 \leq l_{k} \leq d$, also satisfies standard estimates.

The following two lemmas are the analogues of the above two in the case that a $y$ derivative is applied to $q_{1}(f)$. The proof of Lemma 5.16 is significantly different from the proof of Lemma 5.14 so we present it here. However, the proof of Lemma 5.17 is sufficiently close to that of Lemma 5.15 that we omit it.

Lemma 5.16. Suppose that $x \neq y$. If $f \in C^{2}$, then

$$
\begin{aligned}
\frac{x-y}{|x-y|} \cdot \nabla_{y} q_{1}(f) & =-\frac{x-y}{|x-y|^{2}} \cdot \nabla_{y} f(y)+\frac{f(x)-f(y)}{|x-y|^{2}} \\
& =\frac{1}{|x-y|^{2}} \sum_{k=1}^{d}(x-y)_{k} \sum_{j=1}^{d}(x-y)_{j} \int_{0}^{1} b_{j, k}(x, y, t) d t
\end{aligned}
$$

where

$$
b_{j, k}=\int_{0}^{1} \tau \partial_{y_{j}} \partial_{y_{k}} f(t[\tau x+(1-\tau) y]+(1-t) y) d \tau
$$

and $b_{j, k} \in L^{\infty}$.

Proof. The first line of (5.20) is realized by a simple calculation of the derivative of $q_{1}(f)$ with respect to $y$. For the second line we begin with the fundamental theorem of calculus which states that for $f \in C^{1}$,

$$
f(b)-f(a)=\sum_{j=1}^{d} \int_{0}^{1}(b-a)_{j} \partial_{u_{j}} f(t b+(1-t) a) d t .
$$

Provided that $f \in C^{2}$ this can also be done for $\nabla f$ resulting in the following:

$$
\nabla_{u} f(b)-\nabla_{u} f(a)=\sum_{k=1}^{d} \hat{e}_{k} \sum_{j=1}^{d} \int_{0}^{1}(b-a)_{j} \partial_{u_{j}} \partial_{u_{k}} f(t b+(1-t) a) d t
$$

where $\hat{e}_{k}$ is the $k$ th unit vector. We now set $b=\tau x+(1-\tau) y$ and $a=y$, dot with $(x-y)$, and integrate in $\tau$ from 0 to 1 . Using the fundamental theorem of calculus on the $\nabla_{u} f(b)$ term we arrive at

$$
\begin{aligned}
(f(x)- & f(y))-(x-y) \cdot \nabla_{u} f(y) \\
& =\sum_{k=1}^{d}(x-y)_{k} \sum_{j=1}^{d}(x-y)_{j} \int_{0}^{1} \tau \int_{0}^{1} \partial_{u_{j}} \partial_{u_{k}} f(t[\tau x+(1-\tau) y]+(1-t) y) d t d \tau .
\end{aligned}
$$

We now multiply both sides by $\frac{1}{|x-y|^{2}}$ and the theorem is proven. That $b_{j, k}(x, y, t) \in$ $L^{\infty}$ is due to the facts that $f \in C^{2}$ and $\tau \in[0,1]$. 
Lemma 5.17. A Calderón-Zygmund kernel of the type

$$
\zeta(x, y)=k(x, y) \frac{1}{|x-y|^{2}} \sum_{r, s}(x-y)_{r}(x-y)_{s} \prod_{k=1}^{p} \frac{(x-y)_{l_{k}}}{|x-y|}
$$

where $1 \leq r, s, l_{k} \leq d$ and $k(x, y)$ is a Calderón-Zygmund kernel satisfying standard estimates, also satisfies standard estimates.

At this point we can prove both Theorem 5.12 and Theorem 5.13. We begin with Theorem 5.12.

Proof of Theorem 5.12. The main idea is to use the theorem of M. Christ and J. Journé [3] on the operator $S_{p}\left(\eta_{1}, \ldots, \eta_{p}\right)$. Define

$$
L(x, y)=K(x, y) \prod_{j=1}^{p} \frac{(x-y) l_{k}}{|x-y|},
$$

with $K$ as defined in the statement of the theorem and $1 \leq l_{k} \leq d$ as in Lemma 5.14. By Lemma 5.14 we can write the kernel of $S_{p}$

$$
K(x, y) \prod_{j=1}^{p} q_{1}\left(\eta_{j}\right)
$$

as a finite sum of terms of the form

$$
L(x, y) \prod_{j=1}^{p} \int_{0}^{1} \partial_{x_{l_{k}}} \eta_{j}(t x+(1-t) y) d t .
$$

By Lemma 5.15 we know that $L(x, y)$ satisfies standard estimates with $c_{L}=3 p c_{K}$ and $\delta_{L}=\min \left(\delta_{K}, 1\right)$. Therefore we can apply the theorem of M. Christ and J. Journé above to each term

$$
\begin{array}{r}
\left\|\int L(x, y) \prod_{j=1}^{p}\left(\int_{0}^{1} \partial_{x_{l_{k}}} \eta_{j}(t x+(1-t) y) d t\right) \xi(y) d y\right\|_{L^{q}} \\
\leq c_{L} p^{N}\left(\prod_{j=1}^{p}\left|\partial_{x_{l_{k}}} \eta_{j}\right|_{L^{\infty}}\right)\|\xi\|_{L^{q}} .
\end{array}
$$

By the definition of the $C^{1}$ norm the theorem is proven.

The proof of Theorem 5.13 is in many ways a corollary of Theorem 5.12 and is presented below.

Proof of Theorem 5.13. The main idea is to use the theorem of M. Christ and J. Journé [3] on the operator $S_{p}$. We begin with the calculation

$$
\begin{aligned}
S_{p} \xi(x) & =\int K(x, y)\left(\prod_{j=1}^{p} q_{1}\left(\eta_{j}\right)\right) \xi(y) d y \\
& =\int K(x, y)\left(\prod_{j=1, j \neq r}^{p} q_{1}\left(\eta_{j}\right)\right) \frac{\eta_{r}(x) \xi(y)-\eta_{r}(y) \xi(y)}{|x-y|} d y
\end{aligned}
$$




$$
\begin{aligned}
= & \int K(x, y)\left(\prod_{j=1, j \neq r}^{p} q_{1}\left(\eta_{j}\right)\right) q_{1}(\xi) \eta_{r}(y) d y \\
& +\int K(x, y)\left(\prod_{j=1, j \neq r}^{p} q_{1}\left(\eta_{j}\right)\right) \frac{\eta_{r}(x) \xi(y)}{|x-y|} d y \\
& -\int K(x, y)\left(\prod_{j=1, j \neq r}^{p} q_{1}\left(\eta_{j}\right)\right) \frac{\eta_{r}(y) \xi(x)}{|x-y|} d y \\
= & I_{1}+I_{2}+I_{3} .
\end{aligned}
$$

Using Theorem 5.12 with the roles of $\eta_{r}$ and $\xi$ interchanged we can estimate the integral $I_{1}$ in appropriate fashion. We note that this estimate requires that $\eta_{j}, \xi \in C^{1}$ for $j \neq r$, and $\eta_{r} \in L^{q}$. The two integrals $I_{2}$ and $I_{3}$ can be handled in the same way as one another, again with the roles of $\eta_{r}$ and $\xi$ switched. We choose $I_{2}$ and begin by pulling $\eta_{r}(x)$ out in front of the integral. The $\eta_{r}(x)$ factor will be estimated using the $L^{\infty}$ norm. Now we are left with a singular integral operator much like that of Theorem 5.12 except that the singular term is too singular. At this point we require the special form of $K$. In the case $K(x, y)=\frac{x-y}{|x-y|^{d+1}}$ we bring the factor $\frac{x-y}{|x-y|}$ out of the integral by using its $L^{\infty}$ bound and thereby reduce to the case of $K(x, y)=\frac{1}{|x-y|^{\mid}}$. In this case we write

$$
\frac{K(x, y)}{|x-y|}=\frac{1}{|x-y|^{d+1}}=\operatorname{div}_{y}\left[\frac{x-y}{|x-y|^{d+1}}\right] .
$$

We now integrate by parts which results in a kernel of the right singularity at the cost of derivatives appearing on the other terms in the integrand. Without loss of generality consider $K(x, y)=\frac{1}{|x-y|^{d}}$; then the integral $I_{2}$ becomes

$$
\begin{aligned}
I_{2}= & \eta_{r}(x) \int \frac{K(x, y)}{|x-y|}\left(\prod_{j=1, j \neq r}^{p} q_{1}\left(\eta_{j}\right)\right) \xi(y) d y \\
= & -\eta_{r}(x)\left\{\int \frac{(x-y)}{|x-y|^{d+1}} \cdot \nabla_{y}\left[\prod_{j=1, j \neq r}^{p} q_{1}\left(\eta_{j}\right)\right] \xi(y) d y\right. \\
& \left.+\int\left(\prod_{j=1, j \neq r}^{p} q_{1}\left(\eta_{j}\right)\right) \frac{(x-y)}{|x-y|^{d+1}} \cdot \nabla_{y}[\xi(y)] d y\right\} \\
= & -\eta_{r}(x)\left\{\sum_{s=1, s \neq r}^{p} \int \frac{1}{|x-y|^{d}}\left(\prod_{j=1, j \neq r, s}^{p} q_{1}\left(\eta_{j}\right)\right) \frac{(x-y)}{|x-y|} \cdot \nabla_{y}\left[q_{1}\left(\eta_{s}\right)\right] \xi(y) d y\right. \\
& \left.+\int \frac{1}{|x-y|^{d}}\left(\prod_{j=1, j \neq r}^{p} q_{1}\left(\eta_{j}\right)\right) \frac{(x-y)}{|x-y|} \cdot \nabla_{y}[\xi(y)] d y\right\} .
\end{aligned}
$$

Now, from Lemmas 5.16 and 5.17 we can estimate these two terms as long as $\xi \in W^{1, q}$, $\eta_{r} \in L^{\infty}$, and $\eta_{j} \in C^{2}$ for $j \neq r$. As mentioned earlier, the term $I_{3}$ can be handled 
analogously with the requirements that $\xi \in L^{\infty}, \eta_{r} \in W^{1, q}$, and $\eta_{j} \in C^{2}$ for $j \neq r$. The estimate in the statement of the theorem is now realized.

As a precursor to estimating the operators $C_{p}(\eta)$ in $W^{s, q}$ norm, we estimate the following simplified operators:

$$
S_{p}(\eta, \ldots, \eta) \xi(x)=\int k(x, y) q_{1}(\eta)^{p} \xi(y) d y .
$$

These will lead to the operator $C_{p}(\eta)$ since the only difference is the presence in $C_{p}(\eta)$ of the analytic function $c_{p}\left(q_{1}(\eta)\right)$ which we will expand in a Taylor series, giving rise to a sum of operators of the form $S_{p}(\eta, \ldots, \eta)$. With this in mind we prove the following theorem.

THEOREM 5.18. If $d=1$, the following estimate holds for $\eta \in W^{s+1, q}$ and $\xi \in W^{s, q}, s>\max \left(\frac{1}{q}, 2\right)$ :

$$
\left\|\partial_{x}^{s} S_{p}(\eta, \ldots, \eta) \xi\right\|_{L^{q}} \leq C\|\eta\|_{W^{s+1, q}}^{p}\|\xi\|_{W^{s, q}} .
$$

If $d=2$, the following estimate holds for $\eta \in W^{s+2, q}$ and $\xi \in W^{s, q}, s>\max \left(\frac{2}{q}, 3\right)$ :

$$
\left\|\partial_{x}^{s} S_{p}(\eta, \ldots, \eta) \xi\right\|_{L^{q}} \leq C\|\eta\|_{W^{s+2, q}}^{p}\|\xi\|_{W^{s, q}} .
$$

In order to prove this theorem we need the following lemma which is proven in W. Craig, U. Schanz, and C. Sulem [7] and D. Nicholls [19].

Lemma 5.19. Let $S_{p}$ be defined as

$$
S_{p}(\eta, \ldots, \eta) \xi(x)=\int K(x, y) q_{1}(\eta)^{p} \xi(y) d y .
$$

Then the lth derivative of this operator is

$$
\partial_{x}^{l} S_{p}(\eta, \ldots, \eta) \xi(x)=\sum_{m=0}^{l} \sum_{\sum \alpha_{k}=m} S_{p}\left(\partial_{x}^{\alpha_{1}} \eta, \ldots, \partial_{x}^{\alpha_{p}} \eta\right) \partial_{x}^{l-m} \xi(x) .
$$

We are now ready to prove Theorem 5.18.

Proof of Theorem 5.18. By Lemma 5.19 we have

$$
\left\|\partial_{x}^{s} S_{p}(\eta, \ldots, \eta) \xi(x)\right\|_{L^{q}} \leq \sum_{m=0}^{s} \sum_{\sum \alpha_{k}=m}\left\|S_{p}\left(\partial_{x}^{\alpha_{1}} \eta, \ldots, \partial_{x}^{\alpha_{p}} \eta\right) \partial_{x}^{s-m} \xi(x)\right\|_{L^{q}} .
$$

Since we wish to avoid using a $C^{s+1}$ derivative we diverge a little from the approach of W. Craig, U. Schanz, and C. Sulem [7] and split the previous sum into three parts. The first will contain all terms where at least one derivative hits the function $\xi$. The second will contain terms with no derivatives on $\xi$ but not all $s$ derivatives on one $\eta$. The final part will contain all terms where all $s$ derivatives are on one of the $\eta$.

Each part will consist of terms of the form

$$
\left\|S_{p}\left(\partial_{x}^{\alpha_{1}} \eta, \ldots, \partial_{x}^{\alpha_{p}} \eta\right) \partial_{x}^{s-m} \xi(x)\right\|_{L^{q}} .
$$

If $m<s$, then at least one derivative hits $\xi$ and we can use Theorem 5.12 to make the estimate

$$
\begin{aligned}
\left\|S_{p}\left(\partial_{x}^{\alpha_{1}} \eta, \ldots, \partial_{x}^{\alpha_{p}} \eta\right) \partial_{x}^{s-m} \xi(x)\right\|_{L^{q}} & \leq C\left(\prod_{j=1}^{p}\left|\partial_{x}^{\alpha_{j}} \eta\right|_{C^{1}}\right)\left\|\partial_{x}^{s-m} \xi\right\|_{L^{q}} \\
& \leq C|\eta|_{C^{1}}^{p-1}\left|\partial_{x}^{m} \eta\right|_{C^{1}}\left\|\partial_{x}^{s-m} \xi\right\|_{L^{q}}
\end{aligned}
$$


The second line comes from the interpolation

$$
\left|\partial_{x}^{\alpha} \eta\right|_{C^{1}} \leq C|\eta|_{C^{1}}^{1-\frac{\alpha}{m}}\left|\partial_{x}^{m} \eta\right|_{C^{1}}^{\frac{\alpha}{m}} .
$$

By being wasteful with derivatives we can estimate this by $C|\eta|_{C^{1}}^{p-1}|\eta|_{C^{s}}\|\eta\|_{W^{s, q}}$. By our choice of $s$ for $d=1$ we can estimate this by $C\|\eta\|_{W^{s+1, q}}^{p}\|\xi\|_{W^{s, q}}$. Similarly, by the choice of $s$ for $d=2$ we can estimate this by $C\|\eta\|_{W^{s+2, q}}^{p^{s+1}}\|\xi\|_{W^{s, q}}$. If we consider $m=s$ but $\alpha_{r} \neq s$, then we make the estimate, based on Theorem 5.12

$$
\begin{aligned}
\left\|S_{p}\left(\partial_{x}^{\alpha_{1}} \eta, \ldots, \partial_{x}^{\alpha_{p}} \eta\right) \xi(x)\right\|_{L^{q}} & \leq C\left(\prod_{j=1}^{p}\left|\partial_{x}^{\alpha_{j}} \eta\right|_{C^{1}}\right)\|\xi\|_{L^{q}} \\
& \leq C|\eta|_{C^{s}}^{p}\|\xi\|_{L^{q}} .
\end{aligned}
$$

Again, by our choice of $s$ we can make the appropriate estimate. Finally, in the case where $\alpha_{r}=s$ we use Theorem 5.13 to make the estimate

$$
\begin{aligned}
\| S_{p}(\eta, \ldots, & \left.\partial_{x}^{s} \eta, \ldots, \eta\right) \xi(x) \|_{L^{q}} \\
\leq & C\left(\prod_{j=1}^{p}\left|\partial_{x}^{\alpha_{j}} \eta\right|_{C^{1}}\right)\|\xi\|_{L^{q}} \\
\leq & C|\eta|_{C^{1}}^{p-1}\left\{|\eta|_{C^{1}}|\xi|_{C^{1}}\left\|\partial_{x}^{s} \eta\right\|_{L^{q}}\right. \\
& +|\eta|_{C^{1}}\left(\left|\partial_{x}^{s} \eta\right|_{L^{\infty}}\left\|\partial_{x} \xi\right\|_{L^{q}}+|\xi|_{L^{\infty}}\left\|\partial_{x}^{s-1} \eta\right\|_{L^{q}}\right) \\
& \left.+(p-1)|\eta|_{C^{2}}\left(\left|\partial_{x}^{s} \eta\right|_{L^{\infty}}\left\|\partial_{x} \xi\right\|_{L^{q}}+|\xi|_{L^{\infty}}\left\|\partial_{x}^{s} \eta\right\|_{L^{q}}\right)\right\} .
\end{aligned}
$$

Once again, without being careful about derivatives one can make the appropriate estimate given the choice of $s$.

Finally, we are in a position to establish the analyticity of the singular integral operators $C_{p}$ in one and two dimensions in the appropriate function spaces.

THEOREM 5.20. If $d=1, \eta \in W^{s+1, q}$, and $s>\max \left(\frac{1}{q}, 2\right)$, then the singular integral operator $C_{p}(\eta)$ is bounded on $W^{s, q}$ and

$$
\left\|C_{p}(\eta) \xi\right\|_{W^{s, q}} \leq C\|\eta\|_{W^{s+1, q}}^{p}\|\xi\|_{W^{s, q}} .
$$

Furthermore, the operator $C_{p}(\eta)$ is analytic as a mapping on $W^{s, q}$ and thus its Taylor series converges in operator norm. If $d=2, \eta \in W^{s+2, q}$, and $s>\max \left(\frac{2}{q}, 3\right)$, then the singular integral operator $C_{p}(\eta)$ is bounded on $W^{s, q}$ and

$$
\left\|C_{p}(\eta) \xi\right\|_{W^{s, q}} \leq C\|\eta\|_{W^{s+2, q}}^{p}\|\xi\|_{W^{s, q}} .
$$

Furthermore, the operator $C_{p}(\eta)$ is analytic as a mapping on $W^{s, q}$ and thus its Taylor series converges in operator norm.

Proof. The idea behind the proof is to expand the analytic function $c_{p}(z)$ in its Taylor series expansion to reduce $C_{p}$ to an infinite sum of operators $S_{p}$ which have the appropriate decay. In brief we write

$$
\begin{aligned}
C_{p}(\eta) \xi(x) & =\int k(x, y) c_{p}\left(q_{1}\right) \xi(y) d y \\
& =\sum_{l=p}^{\infty} \frac{c_{p}^{(l)}(0)}{l !} S_{p}(\eta, \ldots, \eta) \xi(x) .
\end{aligned}
$$


The proof now proceeds in exactly the same manner as in W. Craig, U. Schanz, and C. Sulem [7] or D. Nicholls [19], where we use Theorem 5.18 rather than the $C^{s+1}-W^{s, q}$ estimates of these papers.

For the smoothing integral operators $C_{p, h}$ there is a development which can be followed which is very similar to, though not as delicate as, the one presented above for the singular integral operators $C_{p}$. Due to the choices of $s$ which we will make, the estimates which we need have already been established by W. Craig, U. Schanz, and C. Sulem in two dimensions [7], and in $d$ dimensions by D. Nicholls [19]. We state the result for completeness, and present the corollary which we use.

TheOREM 5.21. Let $p+\rho+\lambda>d$, and suppose that $|\eta|_{L^{\infty}}<h R_{0}$ and $|\eta|_{C^{s}}<\infty$. Then $C_{p, h}$ is bounded from $L^{q}$ to $W^{s, q}$ and

$$
\left\|C_{p, h}(\eta) \xi\right\|_{W^{s, q}}<C|\eta|_{L^{\infty}}^{p-1}|\eta|_{C^{s}}\|\xi\|_{L^{q}} .
$$

Furthermore, the operator $C_{p, h}$ is analytic as a mapping from $L^{q}$ to $W^{s, q}$ in the set

$$
\left\{\left.\eta|| \eta\right|_{L^{\infty}}<h R_{0} \text { and }|\eta|_{C^{s}}<\infty\right\} .
$$

Consequently, $C_{p, h}$ is represented by its Taylor series expansion.

The corollary that we use is the following.

Corollary 5.22. If $d=1, \eta \in W^{s+1, q}$, and $s>\max \left(\frac{1}{q}, 2\right)$, then the smoothing integral operator $C_{p, h}$ is bounded from $L^{q}$ to $W^{s, q}$ and

$$
\left\|C_{p, h}(\eta) \xi\right\|_{W^{s, q}}<C\|\eta\|_{W^{s+1, q}}^{p}\|\xi\|_{L^{q}} .
$$

Furthermore, the operator $C_{p, h}$ is analytic as a mapping from $L^{q}$ to $W^{s, q}$ and thus is represented by its Taylor series expansion. If $d=2, \eta \in W^{s+2, q}$, and $s>\max \left(\frac{2}{q}, 3\right)$, then the smoothing integral operator $C_{p, h}$ is bounded from $L^{q}$ to $W^{s, q}$ and

$$
\left\|C_{p, h}(\eta) \xi\right\|_{W^{s, q}}<C\|\eta\|_{W^{s+2, q}}^{p}\|\xi\|_{L^{q}} .
$$

Furthermore, the operator $C_{p, h}$ is analytic as a mapping from $L^{q}$ to $W^{s, q}$ and thus is represented by its Taylor series expansion.

Acknowledgment. The first author wishes to thank his wife Kristy for her love and support.

\section{REFERENCES}

[1] J.T. BEALE, The existence of cnoidal water waves with surface tension, J. Differential Equations, 31 (1979), pp. 230-263.

[2] H. Beckert And E. Zeidler, Beiträge zur Theorie und Praxis freier Randwertaufgaben, Akademie-Verlag, Berlin, 1971.

[3] M. Christ And J. Journé, Polynomial growth estimates for multilinear singular integral operators, Acta Math., 159 (1987), pp. 51-80.

[4] R. Coifman And Y. Meyer, Nonlinear harmonic analysis and analytic dependence, in AMS Proc. Symp. Pure Math., 43 (1985), pp. 71-78.

[5] W. Craig and M. Groves, Hamiltonian long-wave scaling limits of the water-wave problem, Wave Motion, 19 (1994), pp. 367-389.

[6] W. Craig and D. Haskell, On the orbit space of the harmonic oscillator, in preparation.

[7] W. Craig, U. Schanz, and C. Sulem, The modulational regime of three-dimensional water waves and the Davey-Stewartson system, Ann. Inst. Henri Poincaré Anal. Non Linéaire, 14 (1997), pp. 615-667.

[8] W. Craig and C. Sulem, Numerical simulation of gravity waves, J. Comput. Phys., 108 (1993), pp. $73-83$. 
[9] E. FAdell AND P. RABinowitz, Generalized cohomological index theories for Lie group actions with an application to bifurcation questions for Hamiltonian systems, Invent. Math., 45 (1978), pp. 139-174.

[10] M.D. Groves and A. Mielke, A Spatial Dynamics Approach to Three-Dimensional GravityCapillary Steady Water Waves, preprint, 1999.

[11] J. Hammack, N. SchefFner, And H. Segur, Two dimensional periodic waves in shallow water, J. Fluid Mech., 209 (1989), pp. 567-589.

[12] J. Hammack, D. McCallister, N. Scheffner, and H. Segur, Two dimensional periodic waves in shallow water II: Asymmetric waves, J. Fluid Mech., 285 (1995), pp. 95-122.

[13] M. Jones AND J. TOLAND, The bifurcation and secondary bifurcation of capillary gravity waves, Proc. Royal Soc. London Ser. A, 399 (1985), pp. 391-417.

[14] M. Jones And J. Toland, Symmetry and bifurcation of capillary gravity waves, Arch. Rational Mech. Anal., 96 (1986), pp. 29-53.

[15] T. Levi-Civita, Détermination rigoureuse des ondes permanentes d'ampleur finie, Math. Ann., 93 (1925), pp. 264-314.

[16] P. Milewski and J.B. Keller, Three dimensional water waves, Stud. Appl. Math., 37 (1996), pp. 149-166.

[17] J. Moser, Periodic orbits near an equilibrium and a theorem by Alan Weinstein, Comm. Pure Appl. Math., 29 (1976), pp. 727-747.

[18] D. Nicholls, Traveling water waves: Spectral continuation methods with parallel implementation, J. Comput. Phys., 142 (1998), pp. 224-240.

[19] D. Nicholls, Traveling Gravity Water Waves in Two and Three Dimensions, Ph.D. Diss., Brown University, Providence, RI, 1998.

[20] D. Nicholls and W. Craig, Traveling Gravity Water Waves in Two and Three Dimensions, in preparation.

[21] P. Plotnikov, Solvability of the problem of spatial gravitational waves on the surface of an ideal fluid, Soviet Physics Dokl., 25 (1980), pp. 170-171.

[22] P. Rabinowitz, Variational methods for nonlinear eigenvalue problems, CIME, III ciclo, Varenna, 1974, Edizioni Cremonese, Rome, 1974, pp. 139-195.

[23] J. Reeder and M. Shinbrot, Three dimensional nonlinear wave interaction in water of constant depth, Nonlinear Anal., 5 (1981), pp. 303-323.

[24] J. Reeder And M. Shinbrot, On Wilton ripples II: Rigorous results, Arch. Rational Mech. Anal., 77 (1981), pp. 321-347.

[25] U. Schanz, On the Evolution of Gravity-Capillary Waves in Three Dimensions., Diss., University of Toronto, Toronto, Ontario, 1997.

[26] J. Stoker, Water Waves: The Mathematical Theory and Applications, Interscience, New York, 1957.

[27] D. Struik, Détermination rigoureuse des ondes irrotationnelles périodiques dans un canal à profondeur finie, Math. Ann., 95 (1926), pp. 595-634.

[28] T.Y. Sun, Three-dimensional steady water waves generated by partially localized pressure disturbances, SIAM J. Math. Anal., 24 (1993), pp. 1153-1178.

[29] A. Weinstein, Normal modes for nonlinear hamiltonian systems, Invent. Math., 20 (1973), pp. $47-57$.

[30] V.E. Zakharov, Stability of periodic waves of finite amplitude on the surface of a deep fluid, J. Appl. Mech. Tech. Phys., 9 (1968), pp. 190-194.

[31] E. ZEIDLER, Existenzbeweis für cnoidal waves unter Berücksichtigung der Oberflächen spannung, Arch. Rational Mech. Anal, 41 (1971), pp. 81-107. 